\title{
Price Discovery and Foreign Participation in Korea's Government Bond Cash and Futures Markets
}

\author{
Jaehun $\mathrm{CHOI}^{*}$ \\ Hosung LIM $^{* *}$ \\ Rogelio Jr. MERCADO*** \\ Cyn-Young PARK ${ }^{* * *}$
}

The views expressed in this paper are those of the authors and do not necessarily reflect the views and policies of the Bank of Korea (BOK) and the Asian Development Bank (ADB) or its Board of Governors or the governments they represent. When reporting or citing this paper, the authors names should always be stated explicitly.

BOK and ADB do not guarantee the accuracy of the data induded in this publication and accepts no responsibility for any consequence of their use

By making any designation of or reference to a particular territory or geographic area, or by using the term "country" in this document, BOK and ADB do not intend to make any judgments as to the legal or other status of any territory or area.

\footnotetext{
* Senior Economist, The Bank of Korea (gh4423@bok.or.kr)

** Economist, The Bank of Korea (hosung@bok.or.kr)

*** Graduate Student/Research Associate, Trinity College Dublin (mercador@tcd.ie)

**** Director, Asian Development Bank (cypark@adb.org)

We are grateful for the hel pful comments and suggestions from Woon Gyu Choi, J inho H uh, J in-Su Park, Hyun-J eong Kim, J ong Ku Kang, I nseon H wang, Sungjin Park, Choong Won Park, Eun Yeong Song. We also wish to thank the seminar participants at the Bank of Korea for the mid-term review of this study in November 2014.
} 


\section{Contents}

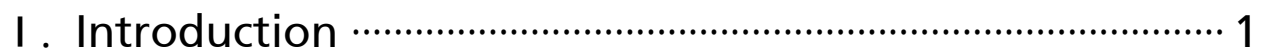

II. Literature Review …………………………………………. 4

III. Dataset, Descriptive Statistics, and Decomposition of Net Purchases and Positions ……………………………... 7

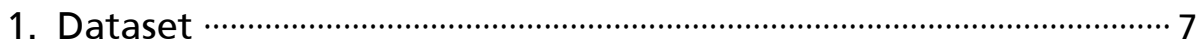

2. Descriptive Statistics ……………………………………………………

3. Decomposition of Net Purchases and Positions ………………... 13

IV. Empirical Specification and Results …….......................2 21

1. Empirical Specification ………………………………………........ 21

2. Analysis of Results ………………………………………………..... 23

V. Summary and Policy Implications ……………………...... 34

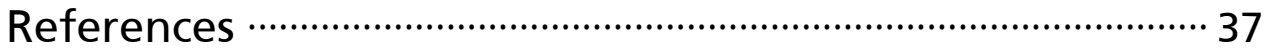




\section{Price Discovery and Foreign Participation in Korea's Government Bond Cash and Futures Markets}

This paper examines the impact of foreign participation in Korean Treasury Bond (KTB) futures and its role in price discovery, using daily transactions data from the over-the-counter market and from the Korea Exchange for the futures. Our analysis suggests that foreign trading in the KTB futures market leads the price discovery process for the underlying bonds. Empirical results show that foreigners' daily net long positions in the futures market exert significant influence in KTB and KTB futures prices. We also find that it is the unexpected component of foreign investors' net long futures positions that explains a significant share of the pricing effects, suggesting that how foreign trading responds to news carries additional information content.

Keywords: Price discovery, Emerging market, Government bond, Foreign participation

JEL Classification: G10, G13, G14 


\section{I . Introduction}

The fast growth of local currency bond markets, combined with the wave of financial globalization, boosted foreign participation in many emerging Asian markets. Local currency bonds outstanding for nine emerging Asian markets reached \$8.0 trillion in September 2014 from about \$0.8 trillion in December 2000, up nearly tenfold. Data from AsianBondsOnline show a dear upward trend in foreign participation across emerging Asian local currency government bond markets since the mid-2000s.1) Growth accelerated even more following a dip in late 2008 associated with the global financial crisis, as emerging Asia's economic resilience, in contrast to the financial turmoil in the United States (US) and the eurozone economies, made their local currency government bonds relatively more attractive to global investors.

Korea has the second largest local currency bond market in emerging Asia,2) with total bonds outstanding at \$1.8 trillion. Starting with the announcement of the Government Bond Market Stimulus Plan in August 1998, a number of policy reforms have been undertaken, induding the introduction of the primary dealer system, interdealer market, and government bond futures in 1999. The Korea Exchange introduced the cash-settled, 3-year Korean Treasury Bond (KTB) futures contract on 29 September 1999.

Foreign holdings of Korean government bonds are now nearly $15 \%$ up from less than $1 \%$ in the mid-2000s. Foreign interest in KTB futures has also been strong, effectively accelerating the growth of the KTB market. Foreigners find it easier to trade futures than cash bonds due to taxation, leverage, and liquidity issues.

Foreign investors have played an important role in the Korean bond markets. Their active participation has helped boost market liquidity, depth, and sophistication. There are also concerns, however, about its potentially destabilizing

1) Data available at $\mathrm{http}: / /$ asianbondsonline.adb.org/regional/data/bondmarket.php?code=Foreign_Holdings

2) Emerging Asia in this paper refers to the nine economies in East and Southeast Asia covering the People's Republic of China; Hong Kong, China; Indonesia; Korea; Malaysia; Philippines; Singapore;, Thailand; and Vietnam. 
effects during financial turmoil. As foreign participation grows, the local bond markets seem to respond more sensitively to global financial conditions as herding behavior among global funds takes hold. Similarly, while the introduction of financial futures facilitates price discovery of the underlying financial assets, some market observers note that greater foreign investor participation in the futures market might raise market volatility with relatively large, one-way transaction volumes compared to those of domestic investors.

The Korean case merits some discussion in this regard. Following the Asian financial crisis, Korean policymakers actively sought the development of domestic bond markets as an alternative source of funding to bank lending. Since then, the market has seen tremendous growth, in no small part due to the establishment of KTB futures and proactive promotion of foreign participation, as many observers note.

But do foreign investors help trigger excessive futures price movements and transmit this instability to the cash market? Price discovery inevitably involves increased market volatility. But if this higher market volatility really only reflects the increase in information as a more heterogeneous groups of investors enters the market, with its diverse sources of information, it should not be a concern in itself. Therefore, the real question should be what type of foreign investors does the futures market attract? Are they different from the type of foreign investors participating in the cash market? And does their trading behavior show more indination toward herding and speculation, leading to excessive market volatility? Better understanding of foreign investors' trading behaviors in futures markets and their role in market efficiency and volatility is critical for assessing the risk of financial liberalization and for designing a macroprudential policy framework appropriate during rapid financial market development.

To our knowledge, no study has looked at the impact on price discovery of sovereign bonds of foreign participation in the futures market. This is especially true for emerging market economies, given a lack of quality trading data. Research in this unexplored area could have a profound impact on financial market development, as the findings could guide policymakers in crafting regulatory guidelines that win the benefits of foreign participation in developing futures markets while avoiding the potentially adverse consequences. This paper 
investigates the price impact of foreign participation in local currency bond futures from the emerging market perspective.

The paper also introduces some important new elements. First, similar to Brandt, Kavajec, and Underwood (2007),3) we examine the trading patterns of different investor groups, specifically looking into the impact of the net transactions of foreign and domestic finance institutions (DFI) on KTB cash and futures and cash prices. We also examine in which market price discovery takes place. Second, following Richards (2005), we decompose net purchases and net long positions into expected and unexpected components to examine how the price discovery happens, but add the analysis of the different types of investor groups to assess whether the type of investor matters in price discovery and, if so, by how much. And third, we look at the discovery process by investor groups at three subperiods - pre-crisis, crisis, and post-crisis - in our sample for any marked changes in the trading behaviors of different investor groups that have influenced price discovery across the subperiods.

Specifically, the paper aims to answer the following three questions:

1. Which market (cash or futures) leads price discovery in Korean treasury bonds?

2. What types of investor groups drive this process?

3. Which component (expected or unexpected) of net purchases and net long positions influences price movements in both markets?

The paper is structured as follows. Section II reviews the related literature. Section III describes our dataset, provides descriptive statistics showing net purchases and net long positions in the cash and futures markets by investor group, and discusses how we decompose expected and unexpected components of net transactions. Section IV presents the empirical model and discusses the results, and section $\mathrm{V}$ summarizes findings and offers policy implications.

3) Brandt, Kavajecz, and Underwood (2007) examine four different investor groups based on whom CBOT members trade for in their accounts. 


\section{Literature Review}

The financial literature on the impact of foreign participation in domestic bond markets is substantial, and suggests that growing foreign participation could influence domestic asset pricing and market liquidity, with potential costs and benefits. Burger, Warnock, and Warnock (2012), International Monetary Fund (2005), Peiris (2010), and Roldos (2004) discuss some of these advantages and disadvantages. Burger, Warnock, and Warnock (2012), for example, daim that foreign participation could support domestic bond market development, as it has the potential to reduce currency mismatches and serve as alternative funding when domestic investors divest. Focusing on emerging markets, Peiris (2010) finds that foreign participation in the domestic government bond market can substantially reduce long-term sovereign bond yields and may even dampen the volatility of bond yields, contrary to a common misconception that foreign participation disturbs the local market. These authors argue that foreign investors can help lower bond yields, increase liquidity in government bond markets, and minimize financial market volatility if supported by institutional and regulatory frameworks. However, they also note that foreign participation could lead to greater interest rate volatility, and induce adverse spillover into other financial markets.

Little has been explored in the role of foreign investors in the government bond price discovery process through their participation in futures trading for emerging economies. But significant evidence points to the interaction of cash and futures markets for treasury bond price discovery in advanced economies. Some empirical work also suggests the importance of investor type for their trading patterns and role in price discovery. To the extent that foreign investors information sets are different than those of domestic investors, foreign participation in emerging financial markets is likely to impact price discovery through various channels of information transmission. It is this gap in the empirical literature we aim to fill using the Korean Treasury bond market as a case study.

A significant body of literature illuminates the role of futures markets in the price discovery of assets in the underlying market. In theory, with perfect information, investors must be indifferent to choices between cash and futures 
markets because new market information is priced in both markets simultaneously. However, empirical evidence often points to substantial price gaps between cash and futures markets, with futures generally leading cash markets, suggesting market inefficiency due to high transaction costs and information barriers between cash and futures markets.

Two aspects of this disconnect between theory and empirics have been studied. The first traces the impact of futures over cash markets or the convergence between cash and futures prices; the second looks at what causes price discovery in the futures market. On the first point, Huang and Zhang (1995) use cointegration and error correction models to assess price discovery between cash and futures municipal bonds in the US, and find that a two-way feedback relationship exists between the two markets, although the feedback from futures to cash markets is the stronger of the two, suggesting price discovery takes place in the futures markets. Campbell and Hendry (2007) use the information-share approach to estimate the contribution of trading in the cash and futures markets to the price discovery in Canadian and US government bonds. These studies on price discovery in advanced countries' sovereign bond markets generally confirm the stylized fact that futures markets lead the cash market in price discovery.

On the second aspect, Kim, Szakmary, and Schwarz (1999) surveyed various explanations of why futures markets lead and they suggest lower trading costs in the futures market as one of the major reasons. The trading cost hypothesis proposed by Fleming, Ostdiek, and Whaley (1996) also suggests that the market with lower overall trading cost will react more instantaneously to new market information, apart from other factors such as differences in market structures, liquidity conditions, short-sale constraints, and regulatory and technical constraints.

In fact, it is widely recognized that exchangetraded futures standardized contracts, and the dearing house, fadilitates price discovery and contributes to relatively low transaction costs. That means relatively low transaction costs in futures markets may give rise to other issues. Some argue that lower transaction costs and margin requirements allow highly leveraged positions and attract speculators who may destabilize the underlying market. Conversely, others argue that futures markets enable speedy price adjustment to new information and attract more informed 
investors, leading to tighter pricing between cash and futures markets, and helping improve liquidity and reduce volatility in the underlying market.

So far, empirical work on price discovery between cash and futures markets has concentrated on equity markets. Theissen (2012), analyzing DAX cash and futures equity prices, finds that the futures market dominates price discovery. Richards (2005) adds another layer of analysis by considering the role played by various investor groups in price discovery between cash and futures equity markets. Specifically, he looks into the impact of foreign participation in both for several Asian economies and finds that foreign investors wishing to change their exposure do so by taking short-term positions in futures markets and then unwinding their futures positions in the subsequent period.

Some note the impact of information or news on bond prices and try to explain the role of trading in price discovery and information/volatility transmission. On the impact of news, Jiang, Lo, and Verdehan (2011) found that while price jumps in US Treasury bonds often occur at prescheduled macroeconomic announcements, news surprises have limited power in explaining bond price jumps. Similarly, Hautsch and Hess (2007) find that the price impact of more precise news (whether good or bad) is stronger than imprecise news.

The market microstructure literature looked at how private information by informed traders can be incorporated into asset prices through order flows. Brandt, Kavajec, and Underwood (2007) look into price discovery in the US Treasury futures markets. They find that although net order flows in both US Treasury cash and futures markets significantly influence prices, types of traders seem to matter for pricing effects in both magnitude and direction. But under illiquid market conditions, price discovery can also take place in the cash market as asymmetric information could be high. Girardi and I mpenna (2013) investigate the role of order flows in price discovery for I talian government bonds, using data from B2B interdealer and B2C dealer-to-customer markets. They note that the explanatory role of order flow is stronger when liquidity conditions are poorer.

This paper builds on Richards (2005) and Brandt, Kavajez, and Underwood (2007) to investigate the role of foreign investors' trading in price discovery using transaction data for KTB and KTB futures markets. 


\section{Dataset, Descriptive Statistics, and Decomposition of Net Purchases and Positions}

\section{Dataset}

We use high quality data on daily net transactions by different investor groups of 3- and 10-year KTBs and their futures. Secondary KTB trading data is collected from the over-thecounter market, while transaction data for KTB futures is from the Korea Exchange. The dataset provides the net purchases and net long positions by investor type of 3- and 10-year KTBs and their futures. First, our analysis focuses on two types of investors -foreign and DFIs. To examine the trading behaviors of different investor types, we disaggregate the types of DFIs into banks, asset management companies, and insurance and pension funds for the cash market; and banks, asset management companies, securities companies, and insurance and pension funds for the futures market.

The data indude a breakdown of net transactions by foreigners and DFIs in daily KTB and KTB futures transactions. Data on foreign net KTB purchases and net positions in futures capture the trading activity of all registered foreign investors. We exdude the domestic securities companies from the DFIs for cash trading data as it is difficult to estimate the correct net purchases data for them.4) We also exduded the transaction data for individual investors, as their participation in secondary cash trading is also quite limited. Because data on futures trading are taken from the Korea Exchange, the net positions of all investor types are of reliable quality.

The dataset runs from $1 \mathrm{~J}$ anuary 2001 to 31 December 2013. However, we limit our period coverage to $1 \mathrm{~J}$ anuary 2004 to 31 December 2013 as the time series of many variables are complete only after 2004, with more active foreign participation in KTB futures. For 3-year cash and futures market, our dataset runs from 1 January 2004 to 31 December 2013. But for 10-year cash and futures, our data

4) Domestic securities companies trade bonds for motivations beyond simple transactions (for example, prenegotiated trading, brokerage, and propriety trading) and as such they can sell KTBs over the counter, while buying them from other sources. 
starts on 25 October 2010, even though the data for 10-year cash is available from 1 J anuary 2004. We use the dean price index5) to compute returns in the cash market, and the dosing price index for futures returns. Non-trading holidays are removed from the data series, while we keep inactive trading days for both markets. For subperiod analysis, we define 1 J anuary 2004 to 15 September 2008 as the precrisis period following the collapse of Lehman Brothers on 15 September 2008. For crisis periods, we chose 16 September 2008 to 31 December 20096), and for post-crisis period 1 J anuary 2010 to 31 December 2013.

Net long positions of different investor groups in the futures markets are expressed as the number of contracts, where each contract has a value of Korean won (KRW) 100,000,000, as are the net purchases of different investor groups in the cash market. Because our dataset is on a daily basis and in contract value, we normalize the daily contract value by the total trading volume for each security. Both 3- and 10-year futures have the same settlement method and cycles.7) For data accuracy, we exdude trading days corresponding to the settlement dates of government bonds (removing returns on both trading and the following days) as the returns on those dates might deviate from normal returns. The final settlement is on a cash basis. At maturity, Korea Exchange settles the difference between the final settlement price and the price of the futures contract.

For the cash markets, 3-year KTB refers to treasury bonds with maturities exceeding 2 years and up to 3 years, and 10-year KTB to maturities exceeding 5 years and up to 10 years. The 3-year KTB futures are more actively traded than the 10-year. I nitially, 10-year futures markets did not grow much because DFIs, especially institutional investors, preferred holding 10-year KTBs until maturity and so hedging demand was limited.8)

5) This is calculated by exduding any interest that has accrued since issuance or the most recent coupon payment (Source: Korea I nvestors Service)

6) We chose 31 December 2009 as the end of the crisis period as the Korean economy recovered from the global economic slowdown in 2010.

7) Settlement dates are in March, J une, September, and December of each year.

8) The underlying asset for the 3-year futures is the 3-year treasury bond, with a face value of KRW100 million and a $5 \%$ coupon rate, while the underlying asset for the 10-year futures is the 10-year treasury bond with a KRW100 million face value and a 5\%coupon rate. 


\section{Descriptive Statistics}

Table 1 (left-side) presents the descriptive statistics of cash and futures returns (mean, standard deviation, skewness, and kurtosis) for both tenors (3- and 10-year) across different sample periods (full sample, pre-crisis, crisis, and post-crisis). The data are computed based on daily logarithmic returns, where non-trading days are excluded. First, for the full sample, futures returns are consistently higher than cash returns although the differences are small. But the differences are generally greater for the 10-year than the 3-year paper, suggesting that the cash and futures markets for 3-year KTBs are better integrated because of greater market liquidity and efficiency given the longer history of trading. Second, futures returns have slightly, but consistently, higher volatility than cash returns. Volatility is also higher for 10-year KTBs and futures compared to the 3-year paper, reflecting its relatively higher yield and perhaps because of limited market liquidity for them. Volatility is also much higher during the crisis period for which data are available for the 3-year KTBs and futures. Third, both cash and futures returns follow a similar distribution pattern, slightly asymmetric and more peaked compared to the normal distribution. Between cash and futures returns of 3-year KTBs, futures returns were more skewed to the left, especially in the pre-crisis period and flatter than the cash returns.

Also table 1 (right-side) presents the trading volume for both cash and futures markets. It reveals that (i) 3-year KTB trading volumes in both cash and futures markets have been rising rapidly, especially futures, with growth after the crisis pronounced; and (ii) for both 3- and 10-year KTBs, futures trading volumes are greater than cash market volumes, reflecting lower transaction costs in the former.

Table 2 presents the average net purchases (cash) and net long positions (futures) by investor group across subperiods. For the cash market, both foreigners and DFIs are net buyers. Because KTB issuance increased over time, all market participants may accumulate net purchases, reflecting market demand growth.

We also find that foreigners increased their net purchases in the 3-year cash market in the crisis and post-crisis periods and reduced their net long positions in the futures market. In the cash market, most DFI subgroups are net buyers, except 
for banks, which are net sellers of 10-year KTBs. We note that foreigners purchased more in the 10-year than the 3-year cash market before the crisis, but this pattern was reversed during the crisis and post-crisis periods. This may be in line with ongoing deleveraging in advanced economies. For the futures market, banks among the DFIs are again the largest players. For 3-year futures, domestic banks, asset management companies, funds, and securities companies are net sellers, although both funds and securities were net buyers during the global financial crisis. For 10-year futures, banks and securities companies are net buyers, while asset management firms and funds are net sellers. Except for banks, other DFIs have smaller positions in the futures market, even compared to foreigners. This supports the view that foreign participation instigates price discovery in the futures markets. Foreigners also show a tendency of herding9) and trend-chasing10) amassing a large net positions compared to DFI s at times.

We also look into how different investor groups react to Bank of Korea (BOK) monetary policy in both cash and futures markets.11) Figures 1 . a and b show the net purchases and net long positions of foreign and DFIs for 3-year government bonds and the futures.12) Foreigners tend to reduce their net long positions in the futures market during BOK monetary policy actions (either easing or tightening stance), leading to the reverse positions of DFI s. In the cash market, DFI sare more active traders than foreigners, although both foreigners and DFI s seem to respond to BOK policy changes by adjusting their net purchases. These results imply that foreign investors who initiate trading activity in the futures market are different from the foreign investors in the cash market and their trading decisions are independent of each other. In addition, DFI sare followers in the futures market by adjusting their net positions as a counterpart to the foreign investors' positions, but they act as an independent major player in the cash market.

9) A similar type of investor following a similar investment strategy may make a common investment decision; for example, simultaneously buying or selling the same securities.

10) Buying finandial securities after a recent upward trend in prices and selling after a recent downward trend. For example, investors can makea consistent one-directional investment for sometime based on their projections for a trend in the policy rate.

11) Policy stance refers to episodes when the BOK increased or decreased the base rate by at least 25 basis points in relation to the previous three interest rate policy decisions.

12) We do not show the 10-year government bonds due to a shorter sample. 


\section{$11 \quad$ BOK Working Paper No.2015-8 (2015.3)}

Table 1: Descriptive Statistics for Returns \& Trading Volume of KTBs

\begin{tabular}{|c|c|c|c|c|c|c|c|c|}
\hline & \multicolumn{4}{|c|}{ Returns } & \multicolumn{4}{|c|}{ Trading Volume } \\
\hline & \multicolumn{2}{|c|}{ 3-Year } & \multicolumn{2}{|c|}{$10-$ Year } & \multicolumn{2}{|c|}{ 3-Year } & \multicolumn{2}{|c|}{ 10-Year } \\
\hline & Cash & Futures & Cash & Futures & Cash & Futures & Cash & Futures \\
\hline \multicolumn{9}{|c|}{ Full sample (01 Jan 2004 to 31 December 2013) } \\
\hline Mean & 0.002 & 0.007 & 0.005 & 0.014 & 25,111 & 158,406 & 29,352 & 71,398 \\
\hline Standard Deviation & 0.14 & 0.16 & 0.27 & 0.32 & 14,413 & 105,153 & 19,116 & 47,535 \\
\hline Skewness & -0.04 & -0.09 & 0.34 & 0.34 & 0.81 & 1.83 & 1.20 & 0.12 \\
\hline Kurtosis & 7.86 & 7.19 & 6.29 & 5.89 & 4.48 & 8.45 & 5.58 & 2.11 \\
\hline Observation & 2186 & 2276 & 754 & 722 & 2,407 & 2,407 & 962 & 766 \\
\hline \multicolumn{9}{|c|}{ Pre-crisis (01 Jan 2004 to 15 September 2008) } \\
\hline Mean & $\begin{array}{r}-0.00 \\
1\end{array}$ & 0.003 & $\ldots$ & $\ldots$ & 18,970 & 92,478 & $\cdots$ & $\cdots$ \\
\hline Standard Deviation & 0.13 & 0.15 & $\cdots$ & $\cdots$ & 11,967 & 47,180 & $\cdots$ & $\ldots$ \\
\hline Skewness & -0.30 & -0.28 & $\cdots$ & $\cdots$ & 1.08 & 1.53 & $\cdots$ & $\cdots$ \\
\hline Kurtosis & 7.21 & 6.61 & $\cdots$ & $\cdots$ & 6.07 & 6.57 & $\cdots$ & $\cdots$ \\
\hline Observation & 975 & 1065 & $\ldots$ & $\ldots$ & 1,130 & 1,130 & $\cdots$ & $\ldots$ \\
\hline \multicolumn{9}{|c|}{ Crisis (16 September 2008 to 31 December 2009) } \\
\hline Mean & 0.005 & 0.016 & $\ldots$ & $\ldots$ & 33,483 & 147,361 & $\cdots$ & $\cdots$ \\
\hline Standard Deviation & 0.24 & 0.26 & $\cdots$ & $\cdots$ & 17,823 & 59,806 & $\cdots$ & $\cdots$ \\
\hline Skewness & -0.02 & -0.11 & $\cdots$ & $\cdots$ & 0.28 & 1.78 & $\cdots$ & $\ldots$ \\
\hline Kurtosis & 4.47 & 4.32 & $\ldots$ & $\cdots$ & 3.41 & 8.10 & $\cdots$ & $\cdots$ \\
\hline Observation & 301 & 301 & $\cdots$ & $\cdots$ & 315 & 315 & $\cdots$ & $\cdots$ \\
\hline \multicolumn{9}{|c|}{ Post-crisis (01 Jan 2010 to 31 December 2013) } \\
\hline Mean & 0.004 & 0.009 & 0.005 & 0.014 & 29,584 & 239,465 & 29,352 & 71,398 \\
\hline Standard Deviation & 0.11 & 0.13 & 0.27 & 0.32 & 12,678 & 110,322 & 19,116 & 47,535 \\
\hline Skewness & 0.34 & 0.19 & 0.34 & 0.34 & 0.84 & 1.80 & 1.20 & 0.12 \\
\hline Kurtosis & 6.88 & 5.74 & 6.29 & 5.89 & 4.88 & 7.92 & 5.58 & 2.11 \\
\hline Observation & 910 & 910 & 754 & 722 & 962 & 962 & 962 & 766 \\
\hline
\end{tabular}

Notes: Values refer to log returns, and 100 mil KRW (Korean won); $\cdots=$ not available.

Table 2: Average Net Purchases and Positions in Cash and Futures Markets

\begin{tabular}{|c|c|c|c|c|c|c|}
\hline & \multicolumn{6}{|c|}{ 3-Year } \\
\hline & Foreigners & $\begin{array}{l}\text { Domestic } \\
\text { Finance } \\
\text { Institutions }\end{array}$ & Banks & $\begin{array}{c}\text { Asset } \\
\text { Management }\end{array}$ & Funds & Securities \\
\hline \multicolumn{7}{|c|}{ Average Net Purchases in Cash Market } \\
\hline Full Sample & 0.700 & 1.149 & 0.733 & 0.143 & 0.273 & $\cdots$ \\
\hline Pre-Crisis & 0.421 & 0.840 & 0.469 & -0.048 & 0.419 & $\cdots$ \\
\hline Crisis & 0.933 & 0.679 & 0.431 & 0.101 & 0.147 & $\cdots$ \\
\hline Post-Crisis & 0.952 & 1.665 & 1.141 & 0.381 & 0.143 & $\cdots$ \\
\hline \multicolumn{7}{|c|}{ Average Net Long positions in Futures Market } \\
\hline Full Sample & 0.089 & -0.068 & -0.038 & -0.009 & -0.006 & -0.015 \\
\hline Pre-Crisis & 0.138 & -0.090 & -0.045 & -0.009 & -0.004 & -0.033 \\
\hline Crisis & 0.031 & -0.058 & -0.083 & -0.006 & 0.001 & 0.033 \\
\hline Post-Crisis & 0.052 & -0.045 & -0.016 & -0.009 & -0.010 & -0.009 \\
\hline
\end{tabular}

Notes: Values in percent of cash and futures trading volumes; $\cdots=$ not available. 
(Table 2, continued)

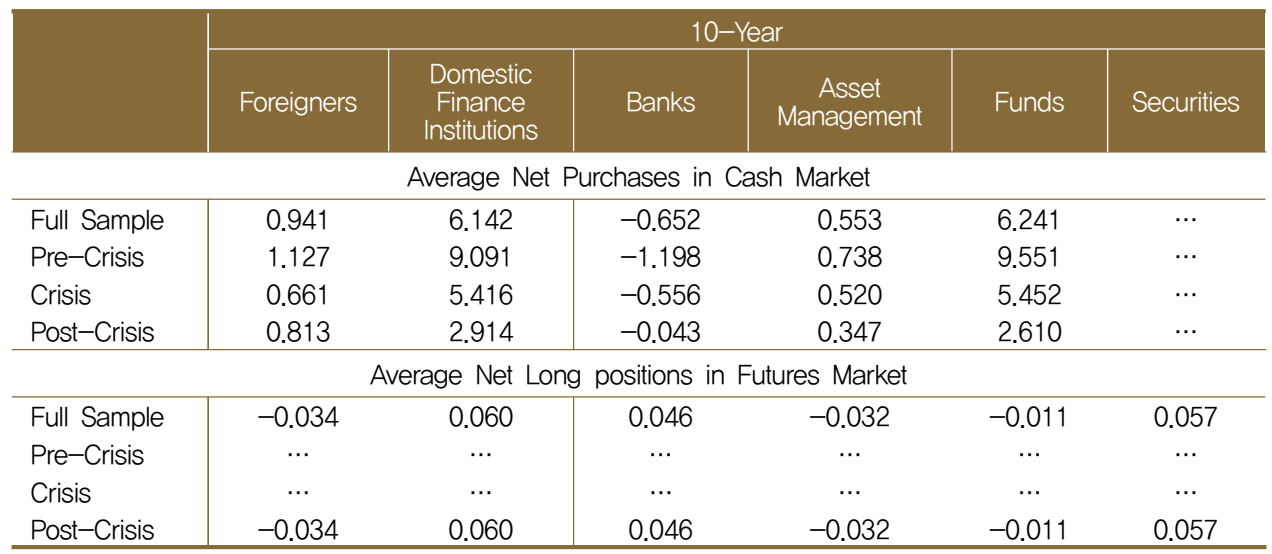

Notes: Values in percent of cash and futures trading volumes; $\cdots=$ not available.

Figure 1: Net Purchases and Positions of Foreign and Domestic Finance Institutions

(a. 3-Year Treasury Cash Market)

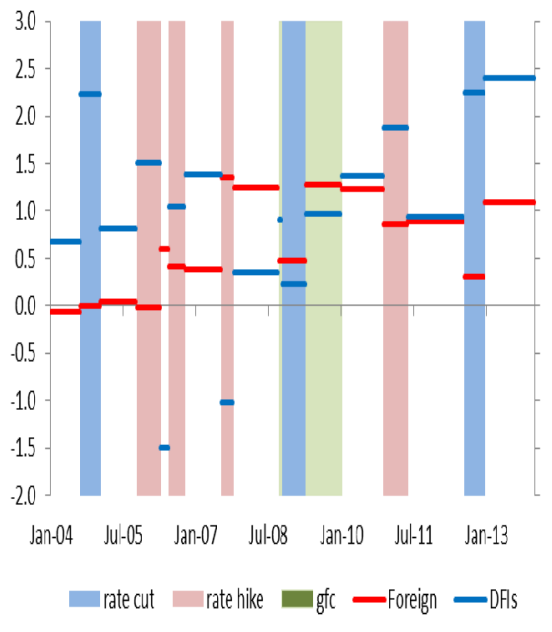

(b. 3-Year Treasury Futures Market)

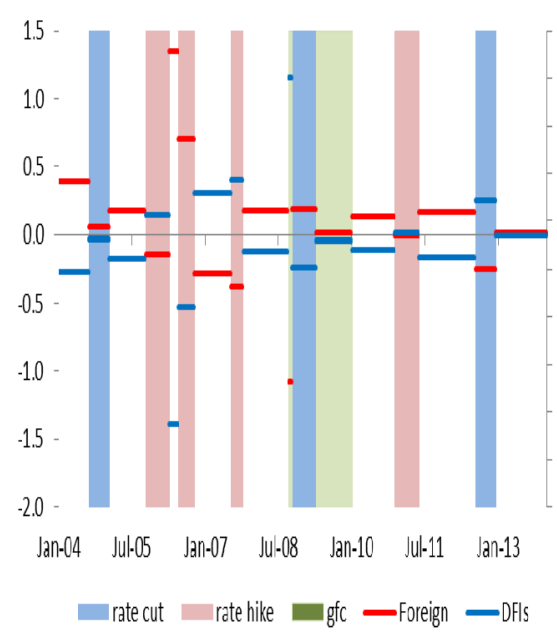

Notes: Futures price index scaled by 100 .

Blue shade $=$ periods of interest rate cuts, red shade $=$ periods of interest hikes, green shade $=$ global financial crisis, DFI = domestic finance institution, gfc = global financial crisis. 


\section{Decomposition of Net Purchases and Positions}

Foreign investors have emerged as important players of increasing influence in the KTB markets, and their trading is dosely watched by other market participants. Do net purchases by foreigners represent additional information to net investor demand in these markets? To understand the price impact of foreign net purchases in KTB cash and futures markets, we try to estimate the new information content of net purchases by foreigners and other investor groups. The trading decisions of foreign investors are presumably based on an information set that may be different from that of domestic investors. Their motivation can be also different from domestic investors.

Richards (2005) presented a model to explain what drives net purchases of foreign investors in emerging market equity and futures. Using a model in which the net purchases of foreign investors are regressed on lagged returns of various markets and other lagged variables such as net flows, he suggests foreign investors tend to respond to price movements in various markets or the information that drives those movements.

Several studies have also looked into the difference between expected and unexpected components of order flows and transaction volumes in financial futures markets. For instance, Bessembinder and Seguin (1993) examined the expected and unexpected components of futures trading volume, and found that unexpected volume shocks have greater impact on volatility. We decompose net purchases into expected and unexpected components. Richards (2005) found both components to be significant. However, the coefficients and variance for the unexpected component are relatively large, suggesting that the majority of the contemporaneous impact on returns can be attributed to the unexpected component.

Following Richards (2005), we decompose the expected and unexpected components by:

$$
\begin{aligned}
N P_{t}= & a+\beta_{1} N P_{t-1}+\beta_{2} N P_{t-2}+\beta_{3} N P_{t-3}+\beta_{4} N P_{t-4}+\beta_{5} N P_{t-5} \\
& +\beta_{6} R E T U R N S_{t-1}+\beta_{7} U S T 10 Y_{t-1}+\beta_{8} K T B 3 Y_{t-1} \\
& +\beta_{9} V I X_{t-1}+\beta_{10} \triangle F X_{t-1}+\varepsilon_{t}
\end{aligned}
$$


Where $N P$ is the net purchases (net long positions) of investor groups, RETURNS $S_{t-1}$ refers to the lagged bond returns (either cash or futures), UST 10Y is the lagged US 10-year Treasury bond yield used as proxy for global interest rates, $K T B 3 Y_{t-1}$ is the lagged yields of 3-year Korean government bonds used as proxy for domestic interest rates, VIX $t_{t-1}$ is the lagged VIX used to measure investor risk appetite, $\Delta \mathrm{FX} \mathrm{T}_{\mathrm{t}-1}$ is the lagged change in nominal exchange rate to account for exchange rate movements, and $\varepsilon$ is the error term. We sourced daily 10-year US Treasury bond yields, 3-year Korean bond yields, nominal exchange rate (the Korean won per US dollar) from the BOK and the VIX from the Chicago Board Options Exchange. We estimate (EQ1) for foreigners and DFIs (including its subgroups) for both cash and futures bond markets.

We derived the unexpected component by estimating (EQ1) and then assuming that the residuals $(\varepsilon t)$ corresponds to the unexpected component, while the expected component corresponds to the fitted values of the above regression. Both expected and unexpected components derived from (EQ1) are also estimated for the three subperiods (pre-crisis, crisis, post-crisis). We do so to account for the changing investor expectations during the three sample periods.

Tables 3a-c present the regression results on the determinants of net purchases and net long-positions for pre-crisis, crisis, and post-crisis periods, respectively. We note several findings. First, lagged net purchases are significant for all sample periods. In the model, the lag length is set at five, as Richards (2005) suggests. ${ }^{13)}$ Net purchases seem to show positive autocorrelation, as investors may build their positions gradually (to mitigate the market impact of their trading) or investors of similar types may respond to new information in similar ways, but with different speeds. Second, net purchases are significantly correlated with lagged returns, which is more pronounced for several investor groups. The lagged returns have significant, negative effects on DFIs' net purchases in the 3-year KTB cash market and on the net long positions of domestic funds and securities companies in the 3-year futures market in the pre-crisis period.

13) We al so tried to fit the model using onelag, but the R-squared was lower. Using five lags improved the model fit. Although the R-squared is low, our model specification seems to beadequate as the residuals do not show any distinct patterns, which may suggest biases in our estimates. 
Table 3a: Determinants of Net Purchases and Net Long Positions (Pre-Crisis Period)

\begin{tabular}{|c|c|c|c|c|c|c|c|c|c|c|c|}
\hline \multirow{2}{*}{ VARIABLES } & (1) & (2) & (3) & (4) & (5) & (6) & (7) & (8) & (9) & (10) & (11) \\
\hline & for3 & fin3 & fin3bk & fin3am & fin3fd & fin3sc & spotfb3 & spotfinb3 & spotfinb3bk & spottinb3am & spotfinb3fd \\
\hline \multirow[t]{2}{*}{$N P(-1)$} & 0.0576 & 0.0639 & 0.0520 & -0.0176 & -0.0482 & 0.0126 & $0.120^{* *}$ & $0.0970^{* *}$ & 0.0626 & $0.149^{* * *}$ & $0.0783^{*}$ \\
\hline & $(0.0502)$ & $(0.0497)$ & $(0.0417)$ & $(0.0453)$ & $(0.0385)$ & $(0.0390)$ & $(0.0590)$ & $(0.0481)$ & $(0.0420)$ & $(0.0367)$ & $(0.0423)$ \\
\hline \multirow[t]{2}{*}{ NP $(-2)$} & $0.122^{* * *}$ & $0.122^{* * *}$ & 0.00360 & $-0.100^{* *}$ & -0.0374 & 0.0349 & 0.0536 & $0.112^{* *}$ & $0.0756^{*}$ & $0.0721^{*}$ & 0.0463 \\
\hline & $(0.0364)$ & $(0.0364)$ & $(0.0393)$ & $(0.0443)$ & $(0.0344)$ & $(0.0348)$ & $(0.0438)$ & $(0.0437)$ & $(0.0445)$ & $(0.0369)$ & $(0.0337)$ \\
\hline \multirow[t]{2}{*}{ NP $(-3)$} & 0.0247 & 0.0287 & -0.0160 & 0.0436 & 0.0157 & 0.000330 & $-0.123^{* *}$ & 0.0133 & -0.0332 & 0.0344 & $0.103^{* * *}$ \\
\hline & $(0.0453)$ & $(0.0439)$ & $(0.0400)$ & $(0.0434)$ & $(0.0414)$ & $(0.0342)$ & $(0.0533)$ & $(0.0367)$ & $(0.0395)$ & $(0.0416)$ & $(0.0327)$ \\
\hline \multirow[t]{2}{*}{ NP $(-4)$} & 0.0427 & 0.0369 & 0.0202 & 0.0101 & -0.0397 & -0.0509 & 0.0385 & -0.0219 & $-0.0768^{* *}$ & -0.0137 & 0.0351 \\
\hline & $(0.0395)$ & $(0.0387)$ & $(0.0363)$ & $(0.0380)$ & $(0.0298)$ & $(0.0396)$ & $(0.0521)$ & (0.0363) & $(0.0369)$ & $(0.0364)$ & $(0.0357)$ \\
\hline \multirow{2}{*}{ NP $(-5)$} & 0.0416 & 0.0204 & $0.0716^{* *}$ & -0.0424 & -0.0234 & 0.0241 & 0.0500 & -0.0520 & $0.0861^{* *}$ & -0.0316 & -0.0552 \\
\hline & $(0.0425)$ & $(0.0409)$ & $(0.0348)$ & (0.0528) & $(0.0442)$ & $(0.0403)$ & $(0.0526)$ & $(0.0420)$ & $(0.0393)$ & $(0.0393)$ & $(0.0392)$ \\
\hline \multirow[t]{2}{*}{ Returns $(-1)$} & 0.860 & -0.497 & 1.040 & $0.584^{*}$ & $-0.272^{* *}$ & $-2.301^{* * *}$ & 0.194 & $-6.253^{* * *}$ & $-2.272^{* *}$ & -1.830 & $-2.195^{* * *}$ \\
\hline & $(1.242)$ & $(1.240)$ & (0.849) & $(0.324)$ & $(0.135)$ & $(0.569)$ & $(0.566)$ & (2.032) & $(1.106)$ & (1.200) & (0.799) \\
\hline \multirow[t]{2}{*}{ UST 3-Yr (-1) } & -0.279 & 0.271 & $0.359^{* *}$ & 0.0167 & 0.0359 & -0.185 & 0.290 & -0.498 & -0.0172 & -0.390 & -0.0899 \\
\hline & $(0.203)$ & $(0.205)$ & $(0.177)$ & $(0.0643)$ & $(0.0290)$ & $(0.116)$ & $(0.215)$ & $(0.386)$ & $(0.289)$ & $(0.250)$ & $(0.169)$ \\
\hline \multirow[t]{2}{*}{ KTB 3-Yr (-1) } & 0.167 & -0.194 & -0.267 & 0.0747 & -0.0809 & 0.195 & -0.147 & -0.217 & -0.239 & 0.254 & -0.207 \\
\hline & $(0.286)$ & $(0.283)$ & $(0.234)$ & $(0.103)$ & $(0.0504)$ & $(0.177)$ & $(0.176)$ & $(0.465)$ & $(0.314)$ & $(0.307)$ & $(0.199)$ \\
\hline \multirow[t]{2}{*}{ VIX $(-1)$} & -0.0213 & 0.0256 & 0.0434 & -0.00209 & 0.00575 & -0.0397 & $0.142^{* * *}$ & -0.0941 & 0.0368 & $-0.105^{*}$ & -0.0177 \\
\hline & $(0.0428)$ & $(0.0424)$ & $(0.0362)$ & $(0.0137)$ & $(0.00620)$ & $(0.0276)$ & $(0.0394)$ & $(0.0841)$ & $(0.0575)$ & $(0.0556)$ & $(0.0334)$ \\
\hline \multirow[t]{2}{*}{$\Delta \mathrm{FX}(-1)$} & 0.453 & $-0.500^{*}$ & -0.0422 & -0.0946 & -0.0366 & -0.213 & -0.00394 & 0.0135 & -0.379 & 0.0137 & $0.333^{*}$ \\
\hline & $(0.282)$ & $(0.277)$ & $(0.224)$ & $(0.105)$ & $(0.0447)$ & $(0.178)$ & $(0.148)$ & $(0.433)$ & $(0.280)$ & $(0.271)$ & $(0.185)$ \\
\hline \multirow[t]{2}{*}{ Constant } & 0.797 & -0.713 & -0.912 & -0.448 & 0.168 & 0.339 & $-2.257^{* * *}$ & $5.107^{* *}$ & 0.984 & 1.958 & $1.891^{* *}$ \\
\hline & (1.209) & $(1.209)$ & (1.023) & $(0.478)$ & $(0.236)$ & $(0.725)$ & $(0.803)$ & $(2.100)$ & (1.405) & (1.390) & $(0.913)$ \\
\hline Observations & 832 & 832 & 832 & 832 & 832 & 832 & 773 & 773 & 773 & 773 & 773 \\
\hline R-squared & 0.033 & 0.031 & 0.016 & 0.020 & 0.014 & 0.028 & 0.080 & 0.062 & 0.030 & 0.051 & 0.055 \\
\hline
\end{tabular}


Table 3b: Determinants of Net Purchases and Net Long Positions (Crisis Period)

\begin{tabular}{|c|c|c|c|c|c|c|c|c|c|c|c|}
\hline \multirow{2}{*}{ VARIABLES } & (1) & (2) & (3) & (4) & (5) & (6) & (7) & (8) & (9) & (10) & (11) \\
\hline & for3 & fin3 & fin3bk & fin3am & fin3fd & fin3sc & spotfb3 & spotfinb3 & spotfinb3bk & spotfinb3am & spotfinb3fd \\
\hline$N P(-1)$ & $\begin{array}{l}0.215^{* * *} \\
(0.0695)\end{array}$ & $\begin{array}{l}0.183^{* * *} \\
(0.0701)\end{array}$ & $\begin{array}{l}-0.00428 \\
(0.0595)\end{array}$ & $\begin{array}{l}0.0191 \\
(0.0721)\end{array}$ & $\begin{array}{l}-0.0279 \\
(0.0707)\end{array}$ & $\begin{array}{l}0.0437 \\
(0.0621)\end{array}$ & $\begin{array}{l}0.236^{* *} \\
(0.108)\end{array}$ & $\begin{array}{l}0.0831 \\
(0.0507)\end{array}$ & $\begin{array}{l}-0.0601 \\
(0.0707)\end{array}$ & $\begin{array}{l}0.0385 \\
(0.0633)\end{array}$ & $\begin{array}{l}0.0468 \\
(0.0642)\end{array}$ \\
\hline$N P(-2)$ & $\begin{array}{l}0.107^{*} \\
(0.0630)\end{array}$ & $\begin{array}{l}0.134^{* *} \\
(0.0573)\end{array}$ & $\begin{array}{l}0.0624 \\
(0.0546)\end{array}$ & $\begin{array}{l}-0.0224 \\
(0.0553)\end{array}$ & $\begin{array}{l}0.0466 \\
(0.0744)\end{array}$ & $\begin{array}{l}-0.0170 \\
(0.0565)\end{array}$ & $\begin{array}{l}-0.0318 \\
(0.0618)\end{array}$ & $\begin{array}{l}-0.0411 \\
(0.0504)\end{array}$ & $\begin{array}{l}-0.0130 \\
(0.0623)\end{array}$ & $\begin{array}{l}-0.0158 \\
(0.0672)\end{array}$ & $\begin{array}{l}-0.0241 \\
(0.0758)\end{array}$ \\
\hline$N P(-3)$ & $\begin{array}{l}-0.0335 \\
(0.0607)\end{array}$ & $\begin{array}{l}-0.0416 \\
(0.0574)\end{array}$ & $\begin{array}{l}0.00241 \\
(0.0553)\end{array}$ & $\begin{array}{l}-0.00906 \\
(0.0551)\end{array}$ & $\begin{array}{l}-0.123^{*} \\
(0.0738)\end{array}$ & $\begin{array}{l}-0.0157 \\
(0.0640)\end{array}$ & $\begin{array}{l}0.0297 \\
(0.0549)\end{array}$ & $\begin{array}{l}-0.00465 \\
(0.0595)\end{array}$ & $\begin{array}{l}-0.0779 \\
(0.0596)\end{array}$ & $\begin{array}{l}0.0655 \\
(0.0844)\end{array}$ & $\begin{array}{l}-0.0735 \\
(0.0637)\end{array}$ \\
\hline$N P(-4)$ & $\begin{array}{l}0.151^{* *} \\
(0.0616)\end{array}$ & $\begin{array}{l}0.163^{* * *} \\
(0.0611)\end{array}$ & $\begin{array}{l}-0.104 \\
(0.0664)\end{array}$ & $\begin{array}{l}0.124^{* * *} \\
(0.0454)\end{array}$ & $\begin{array}{l}0.134^{*} \\
(0.0754)\end{array}$ & $\begin{array}{l}-0.0444 \\
(0.0653)\end{array}$ & $\begin{array}{l}0.100 \\
(0.0721)\end{array}$ & $\begin{array}{l}0.0338 \\
(0.0604)\end{array}$ & $\begin{array}{l}0.00252 \\
(0.0537)\end{array}$ & $\begin{array}{l}-0.0201 \\
(0.0679)\end{array}$ & $\begin{array}{l}0.0870 \\
(0.0635)\end{array}$ \\
\hline$N P(-5)$ & $\begin{array}{l}0.0569 \\
(0.0638)\end{array}$ & $\begin{array}{l}0.0549 \\
(0.0632)\end{array}$ & $\begin{array}{l}0.0227 \\
(0.0626)\end{array}$ & $\begin{array}{l}0.0636 \\
(0.0497)\end{array}$ & $\begin{array}{l}0.0623 \\
(0.0649)\end{array}$ & $\begin{array}{l}0.131^{*} \\
(0.0675)\end{array}$ & $\begin{array}{l}0.0624 \\
(0.0641)\end{array}$ & $\begin{array}{l}0.103^{*} \\
(0.0545)\end{array}$ & $\begin{array}{l}0.0501 \\
(0.0610)\end{array}$ & $\begin{array}{l}-0.0202 \\
(0.0576)\end{array}$ & $\begin{array}{l}-0.00806 \\
(0.0628)\end{array}$ \\
\hline Returns $(-1)$ & $\begin{array}{l}0.921 \\
(0.705)\end{array}$ & $\begin{array}{l}-0.820 \\
(0.721)\end{array}$ & $\begin{array}{l}-1.340^{* *} \\
(0.540)\end{array}$ & $\begin{array}{l}-0.462^{* * *} \\
(0.172)\end{array}$ & $\begin{array}{l}0.0924 \\
(0.125)\end{array}$ & $\begin{array}{l}0.595 \\
(0.476)\end{array}$ & $\begin{array}{l}-0.872^{*} \\
(0.484)\end{array}$ & $\begin{array}{l}1.149 \\
(1.142)\end{array}$ & $\begin{array}{l}1.175 \\
(0.834)\end{array}$ & $\begin{array}{l}-0.327 \\
(0.611)\end{array}$ & $\begin{array}{l}-0.255 \\
(0.458)\end{array}$ \\
\hline UST 3-Yr (-1) & $\begin{array}{l}-1.911^{* *} \\
(0.927)\end{array}$ & $\begin{array}{l}1.847^{* *} \\
(0.917)\end{array}$ & $\begin{array}{l}0.551 \\
(0.743)\end{array}$ & $\begin{array}{l}0.349^{*} \\
(0.186)\end{array}$ & $\begin{array}{l}0.174 \\
(0.214)\end{array}$ & $\begin{array}{l}1.411^{* *} \\
(0.700)\end{array}$ & $\begin{array}{l}-0.663 \\
(0.640)\end{array}$ & $\begin{array}{l}1.540 \\
(1.337)\end{array}$ & $\begin{array}{l}0.669 \\
(1.003)\end{array}$ & $\begin{array}{l}0.389 \\
(0.483)\end{array}$ & $\begin{array}{l}1.521^{* *} \\
(0.620)\end{array}$ \\
\hline KTB 3-Yr (-1) & $\begin{array}{l}0.491 \\
(0.424)\end{array}$ & $\begin{array}{l}-0.553 \\
(0.416)\end{array}$ & $\begin{array}{l}-0.127 \\
(0.360)\end{array}$ & $\begin{array}{l}-0.0650 \\
(0.0989)\end{array}$ & $\begin{array}{l}-0.0535 \\
(0.102)\end{array}$ & $\begin{array}{l}-0.286 \\
(0.323)\end{array}$ & $\begin{array}{l}0.340 \\
(0.343)\end{array}$ & $\begin{array}{l}-1.018 \\
(0.650)\end{array}$ & $\begin{array}{l}-0.515 \\
(0.494)\end{array}$ & $\begin{array}{l}-0.340 \\
(0.518)\end{array}$ & $\begin{array}{l}-0.584^{* *} \\
(0.273)\end{array}$ \\
\hline $\operatorname{VIX}(-1)$ & $\begin{array}{l}-0.00297 \\
(0.0118)\end{array}$ & $\begin{array}{l}0.00376 \\
(0.0116)\end{array}$ & $\begin{array}{l}0.00682 \\
(0.00924)\end{array}$ & $\begin{array}{l}0.000843 \\
(0.00313)\end{array}$ & $\begin{array}{l}-0.00206 \\
(0.00306)\end{array}$ & $\begin{array}{l}-0.00170 \\
(0.00842)\end{array}$ & $\begin{array}{l}-0.0191^{* *} \\
(0.00936)\end{array}$ & $\begin{array}{l}-0.00723 \\
(0.0178)\end{array}$ & $\begin{array}{l}0.00691 \\
(0.0131)\end{array}$ & $\begin{array}{l}-0.00155 \\
(0.0108)\end{array}$ & $\begin{array}{l}-0.00872 \\
(0.00698)\end{array}$ \\
\hline$\Delta F X(-1)$ & $\begin{array}{l}0.120^{*} \\
(0.0660)\end{array}$ & $\begin{array}{l}-0.155^{* *} \\
(0.0688)\end{array}$ & $\begin{array}{l}-0.0731 \\
(0.0770)\end{array}$ & $\begin{array}{l}0.0159 \\
(0.0263)\end{array}$ & $\begin{array}{l}-0.0113 \\
(0.0212)\end{array}$ & $\begin{array}{l}-0.0584 \\
(0.0668)\end{array}$ & $\begin{array}{l}-0.0442 \\
(0.0664)\end{array}$ & $\begin{array}{l}0.0154 \\
(0.143)\end{array}$ & $\begin{array}{l}0.0435 \\
(0.145)\end{array}$ & $\begin{array}{l}0.0436 \\
(0.0666)\end{array}$ & $\begin{array}{l}-0.0185 \\
(0.0611)\end{array}$ \\
\hline Constant & $\begin{array}{l}0.991 \\
(1.022)\end{array}$ & $\begin{array}{l}-0.684 \\
(1.059)\end{array}$ & $\begin{array}{l}-0.715 \\
(1.103)\end{array}$ & $\begin{array}{l}-0.281 \\
(0.250)\end{array}$ & $\begin{array}{l}0.0416 \\
(0.279)\end{array}$ & $\begin{array}{l}-0.810 \\
(0.926)\end{array}$ & $\begin{array}{l}0.817 \\
(1.126)\end{array}$ & $\begin{array}{l}2.711 \\
(2.181)\end{array}$ & $\begin{array}{l}1.436 \\
(1.684)\end{array}$ & $\begin{array}{l}0.234 \\
(1.544)\end{array}$ & $\begin{array}{l}0.587 \\
(0.780)\end{array}$ \\
\hline Observations & 253 & 253 & 253 & 253 & 253 & 253 & 253 & 253 & 253 & 253 & 253 \\
\hline R-squared & 0.187 & 0.178 & 0.052 & 0.068 & 0.052 & 0.048 & 0.129 & 0.037 & 0.028 & 0.017 & 0.063 \\
\hline
\end{tabular}

Notes: for $=$ foreign futures, FIN $=$ DFIs futures, $b k=$ DFI banks futures/spot, am = DFI asset management futures/spot, fd = DFI funds futures/spot, $s c=D F I$ securities futures, spotfb = foreign spot, spotfin = DFI spot; Robust standard errors in parentheses. ${ }^{* * *} p<0.01,{ }^{* *} p<0.05,{ }^{*} p<0.1$ 
Table 3c: Determinants of Net Purchases and Net Long Positions (Post-Crisis Period)

\begin{tabular}{|c|c|c|c|c|c|c|c|c|c|c|c|c|c|c|c|c|c|c|c|c|c|c|}
\hline \multirow{2}{*}{ VARIABLES } & (1) & (2) & (3) & (4) & (5) & (6) & (7) & (8) & (9) & (10) & (11) & (12) & (13) & (14) & (15) & (16) & (17) & (18) & (19) & (20) & (21) & (22) \\
\hline & for3 & fin 3 & fin $3 b k$ & fin3am & fin $3 f d$ & fin $3 s c$ & spotitb3 & spotinib3 & spotifin33k & spotinibam & spodinb33d & for10 & fin10 & fin10bk & fin10am & fin10id & fin $10 \mathrm{sc}$ & spottb10 & spodirnb10 | & spotinblodk & spodiribilom & spodirbiold \\
\hline \multirow[t]{2}{*}{$N P(-1)$} & $0.300^{4 * 4 *}$ & $0.300^{4 * * *}$ & -0.0525 & 0.00572 & $-0.153^{* *}$ & $0.12^{* 4 * *}$ & 0.128 & $0.0813^{*}$ & 0.0619 & $0.168^{\text {two }}$ & 0.0246 & 0.0842 & 0.0541 & -0.119 & 0.0270 & 0.0610 & $-0.246 *$ & $0.160^{* *}$ & $0.178^{* *}$ & -0.0529 & $0.108^{* * * *}$ & $0.196^{* * * *}$ \\
\hline & $(0.0493)$ & $(0.0491)$ & $(0.0372)$ & $(0.0451)$ & $(0.0634)$ & $(0.0433)$ & $(0.0982)$ & $(0.0427)$ & $(0.0390)$ & $(0.0377)$ & $(0.0869)$ & $(0.0806)$ & $(0.0797)$ & $(0.0785)$ & $(0.0584)$ & $(0.114)$ & $(0.0639)$ & $(0.0699)$ & $(0.0694)$ & $(0.0482)$ & $(0.0402)$ & $(0.0662)$ \\
\hline \multirow[t]{2}{*}{$N P(-2)$} & $0.122^{* k *}$ & $0.113^{* *}$ & $-00025^{*}$ & 0.0316 & -0.0408 & 0.0451 & -0.00525 & 0.0476 & 0.0221 & $0.0710^{*}$ & 0.0217 & -0.0327 & -0.0203 & -0.097 & 0.109 & 0.0601 & -0.0905 & 0.0628 & $0.149^{* *}$ & 0.0536 & 0.0497 & 0.0887 \\
\hline & $(0.0456)$ & $(0.0458)$ & $(0.0372)$ & $(0.0416)$ & $(0.0579)$ & $(0.0393)$ & $(0.0538)$ & $(0.0382)$ & $(0.0367)$ & $(0.0418)$ & $(0.0364)$ & $(0.151)$ & $(0.132)$ & $(0.0085)$ & $(0.0699)$ & $(0.0861)$ & $(0.0644)$ & $(0.0099)$ & $(0.0614)$ & $(0.0533)$ & $(0.0474)$ & $(0.0732)$ \\
\hline \multirow[t]{2}{*}{$N P(-3)$} & $0.117^{7 * * *}$ & $0.115^{* * *}$ & -0.0227 & 0.00414 & -0.00124 & 0.0417 & 0.0139 & 0.0368 & 0.00483 & $0.102^{* *}$ & -0.00898 & 0.0363 & 0.0779 & 0.00539 & -0.00580 & -0.0440 & -0.0140 & $0.166^{* k * *}$ & 0.00338 & $0.0780^{*}$ & -0.00061 & 0.0003 \\
\hline & $(0.0425)$ & $(0.0424)$ & $(0.0370)$ & $(0.0358)$ & $(0.0563)$ & $(0.0430)$ & $(0,0263)$ & $(0.0451)$ & $(0.0372)$ & $(0.0411)$ & $(0.0398)$ & $(0.0495)$ & $(0.0597)$ & $(0.0820)$ & $(0.0564)$ & $(0.0740)$ & $(0.0006)$ & $(0.0561)$ & $(0.0408)$ & $(0.0455)$ & $(0.0419)$ & $(0.0700)$ \\
\hline \multirow[t]{2}{*}{$N P(-4)$} & 0.00275 & 0.0107 & -0.0211 & -0.0601 & -0.0348 & $0.0658^{*}$ & 0.0159 & -0.00067 & -0.0223 & -0.00682 & 0.0699 & -0.138 & -0.125 & -0.0234 & 0.0445 & -0.0870 & -0.0252 & 0.0178 & 0.0276 & $0.0554^{* *}$ & -0.0101 & -0.0506 \\
\hline & $(0.0407)$ & $(0.0406)$ & $(0.0385)$ & $(0.0429)$ & $(0.0482)$ & $(0.0395)$ & $(0.0272)$ & $(0.0391)$ & $(0.0331)$ & $(0.0357)$ & $(0.0459)$ & $(0.131)$ & $(0.117)$ & $(0.0638)$ & $(0.0346)$ & $(0.0742)$ & $(0.0596)$ & $(0.0513)$ & $(0.0447)$ & $(0.0421)$ & $(0.0611)$ & $(0.0531)$ \\
\hline \multirow[t]{2}{*}{$N P(-5)$} & $-0.08090^{* *}$ & $-0.0667^{* *}$ & -0.000215 & 0.00679 & 0.0548 & -0.0353 & 0.0207 & -0.00007 & -0.0378 & -0.0054 & -0.0441 & 0.0137 & -0.0326 & -0.0368 & 0.0334 & 0.117 & -0.0619 & 0.0672 & -0.0294 & -0.0315 & -0.0363 & $0.0942^{*}$ \\
\hline & $(0.0392)$ & $(0.0390)$ & $(0.0071)$ & $(0.0367)$ & $(0.0484)$ & $(0.0384)$ & $(0.0311)$ & $(0.0401)$ & $(0.0349)$ & $(0.0420)$ & $(0.0465)$ & $(0.0428)$ & $(0.0513)$ & $(0.0845)$ & $(0.0548)$ & $(0.0974)$ & $(0.0635)$ & $(0.0414)$ & $(0.0422)$ & $(0.0472)$ & $(0.0398)$ & $(0.0568)$ \\
\hline \multirow[t]{2}{*}{ Returns $(-1)$} & $-1.655^{* *}$ & $1.656^{* *}$ & $-1.462^{* * * *}$ & $0.242^{*}$ & -0.211 & $1.844^{\text {pak }}$ & 0.750 & -2.207 & -1.998 & 0.280 & -0.512 & -0.232 & 0.170 & 0.403 & $0.113^{* *}$ & 0.0110 & -0.265 & 0.292 & -0.280 & 0.436 & 0.136 & -0.559 \\
\hline & $(0.726)$ & $(0.709)$ & (0.530) & $(0.143)$ & $(0.200)$ & $(0.698)$ & $(0.991)$ & (2011) & $(1,398)$ & $(0.868)$ & $(0.750)$ & $(0.192)$ & $(0.226)$ & $(0.351)$ & $(0.0558)$ & $(0.111)$ & $(0.347)$ & $(0.283)$ & $(0.895)$ & $(0.515)$ & $(0.215)$ & $(0.855)$ \\
\hline \multirow[t]{2}{*}{ UST 3-Yr (-1) } & 0.337 & -0.302 & $-0.542^{*}$ & -0.0228 & -0.0226 & 0.145 & 0.523 & 0.689 & 0.807 & 0.230 & -0.274 & -0.277 & 0.363 & $0.475^{*}$ & 0.0100 & $0.129^{* *}$ & -0.144 & -0.184 & 0.778 & -0.291 & -0.0428 & 0.980 \\
\hline & $(0.385)$ & $(0.381)$ & $(0,298)$ & $(0.0638)$ & $(0.147)$ & $(0.386)$ & $(0.374)$ & $(0.828)$ & (0.568) & $(0.312)$ & $(0.315)$ & $(0.217)$ & $(0.224)$ & $(0,261)$ & $(0.0334)$ & $(0.0644)$ & $(0.247)$ & $(0.179)$ & $(0.671)$ & $(0.375)$ & $(0.142)$ & $(0.643)$ \\
\hline \multirow[t]{2}{*}{ KTB 3-Yr (-1) } & -0.0633 & 0.0674 & $0.494^{*}$ & -0.0656 & -0.123 & -0.286 & -0.497 & $-1.670^{*}$ & $-1.549^{* * *}$ & -0.358 & 0.203 & 0.477 & $-0.515^{*}$ & 0.00744 & -0.0986 & $-0.415^{* *}$ & -0.0699 & 0.323 & 0.789 & 0.348 & $0.634^{* *}$ & -0.254 \\
\hline & $(0.384)$ & $(0.377)$ & $(0.267)$ & $(0.0597)$ & $(0.132)$ & $(0.384)$ & $(0.356)$ & $(0.852)$ & (0.588) & $(0.325)$ & $(0.313)$ & $(0.298)$ & $(0.305)$ & $(0.409)$ & $(0.0624)$ & $(0.193)$ & $(0.413)$ & $(0.303)$ & $(1.116)$ & $(0.612)$ & $(0.246)$ & $(1.140)$ \\
\hline \multirow[t]{2}{*}{$\operatorname{VIX}(-1)$} & 0.0247 & -0.0236 & -0.01000 & -0.00190 & 0.00154 & -0.00141 & 0.0293 & -0.0579 & 0.00145 & -0.0137 & $-0.0502^{* * * *}$ & -0.0155 & 0.0160 & -0.00856 & 0.00156 & $0.0370^{*}$ & -0.0168 & -0.0166 & -0.0480 & -0.0186 & -0.0108 & -0.00935 \\
\hline & $(0.0160)$ & $(0.0161)$ & $(0.0138)$ & $(0.00001)$ & $(0.00687)$ & $(0.0167)$ & $(0.0303)$ & $(0.0379)$ & $(0.0309)$ & $(0.0158)$ & $(0.0143)$ & $(0.0132)$ & $(0.0137)$ & $(0.0190)$ & $(0.00396)$ & $(0.0191)$ & $(0.0266)$ & $(0.0141)$ & $(0.0531)$ & $(0.0303)$ & $(0.0131)$ & $(0.0475)$ \\
\hline \multirow[t]{2}{*}{$\Delta \mathrm{FX}(-1)$} & 0.182 & -0.200 & 0.104 & $-0.0462^{*}$ & -0.0542 & $-0,231^{*}$ & $-0.45^{4+4 k}$ & -0.460 & -0.304 & -0.0419 & -0.119 & 0.0294 & -0.0358 & 0.324 & -0.00893 & -0.0468 & -0.335 & -0.0511 & $-1.420^{\text {kw* }}$ & $-0.694^{* *}$ & -0.0329 & -0.606 \\
\hline & $(0.151)$ & $(0.154)$ & $(0.110)$ & $(0.0274)$ & $(0.0479)$ & $(0.139)$ & $(0.173)$ & $(0.322)$ & $(0.233)$ & $(0.133)$ & $(0.130)$ & $(0.126)$ & $(0.140)$ & $(0.221)$ & $(0.0459)$ & $(0.106)$ & $(0,254)$ & $(0.176)$ & $(0.485)$ & $(0.279)$ & $(0.0897)$ & $(0.382)$ \\
\hline \multirow[t]{2}{*}{ Constant } & -0.434 & 0.380 & -0.476 & $0.253^{*}$ & 0.376 & 0.849 & $1.448^{*}$ & $7.66^{-3 * 4}$ & $5.703^{* * * *}$ & $1.569^{*}$ & 0.650 & -0.635 & 0.570 & -0.840 & 0.230 & 0.358 & 0.856 & -0.0380 & -1.762 & -0.401 & $-1.425^{* * *}$ & 0.186 \\
\hline & $(0.982)$ & $(0.964)$ & $(0.611)$ & $(0.144)$ & $(0.252)$ & $(0.963)$ & $(0.868)$ & (2218) & (1.576) & $(0.835)$ & $(0.764)$ & $(0.554)$ & $(0.584)$ & $(0.940)$ & $(0.160)$ & $(0.382)$ & $(0.986)$ & $(0.619)$ & (2061) & $(1.196)$ & $(0.492)$ & (1.973) \\
\hline Observations & 720 & 720 & 720 & 720 & 720 & 720 & 720 & 720 & 720 & 720 & 720 & 564 & 564 & 564 & 564 & 564 & 564 & 595 & 595 & 595 & 595 & 595 \\
\hline R-squared & 0.157 & 0.151 & 0.038 & 0.022 & 0.034 & 0.034 & 0.032 & 0.048 & 0.028 & 0.061 & 0.029 & 0.031 & 0.032 & 0.043 & 0.036 & 0.048 & 0.078 & 0.096 & 0.111 & 0.036 & 0.044 & 0.114 \\
\hline
\end{tabular}

Notes: for $=$ foreign futures, FIN $=$ DFls futures, bk $=$ DFI banks futures/spot, am $=$ DFI asset management futures/spot, fd $=$ DFI funds futures/spot, $s$

$s c=$ DFI securities futures, spotfb $=$ foreign spot, spotfin $=$ DFI spot; $\quad$ Robust standard errors in parentheses. ${ }^{* *} p<0.01, * * p<0.05, * p<0.1$ 
The effects of lagged returns on net purchases in the cash market become rather insignificant in the crisis and post-crisis periods, but the lagged returns have significant effects on net long positions of foreign investors in the futures market. The negative coefficients in the cash market suggest lower returns lead to higher net purchases, as opposed to the finding in Richards (2005). This may suggest that there is some underlying demand for KTBs attracting investors to buy when the price falls. In the earlier years, DFIs have increased their holdings of KTBs for various reasons other than pure investment purposes. This pattern has changed over time, as their KTB holdings may have reached adequate levels. On the other hand, the effects of lagged returns have become significant in the 3-year futures market, with foreigners buying on previous day low returns. In the post-crisis period, the effects of lagged returns are more significant for 3- than for 10-year KTBs, implying that for long-term investors, daily returns may not be as important as they are for short-term investors. Third, the muted effects of lagged returns in more recent periods may al so suggest that investors extract information from more broadly based information sets and the lagged returns may provide only little new information. What is noticeable is that the explanatory power of other information variables such as US and Korean interest rates, risk aversion, and exchange rate changes has become more significant over time. This could imply that investors extract more information from global and domestic economic conditions and potential risk factors for their trading decisions.

Table 4 shows the summary statistics for the expected component of net purchases, the fitted values derived from the above regression results. Overall, we find that net purchases in the cash markets are better fitted by the model than the net long positions in the futures, with explanatory variables induding lagged net purchases, lagged returns, and other economic variables. The model seems to explain DFIs' net purchases in the cash market better than foreign investors' net purchases. In the futures market, the net positions of both foreign and domestic investors seem to be driven by news or private information, as suggested by the relatively low value of the expected component. Foreign investors and DFI groups take the opposite positions in the futures market. It seems foreign investors have been net buyers of 3-year futures contracts, while DFI s have been net sellers. 
Table 4: Expected Component of Net Purchases and Positions by Investor Groups

\begin{tabular}{|c|c|c|c|c|c|c|c|c|c|c|}
\hline \multirow{3}{*}{ Variable } & \multicolumn{10}{|c|}{ 3-year KTB } \\
\hline & \multicolumn{5}{|c|}{ Pre-crisis } & \multicolumn{5}{|c|}{ Crisis } \\
\hline & Obs & Mean & Std. Dev. & Min & Max & Obs & Mean & Std. Dev. & Min & Max \\
\hline Foreign Futures & 867 & 0.29 & 0.74 & -2.83 & 2.90 & 259 & 0.24 & 1.16 & -3.93 & 2.69 \\
\hline DFI Futures & 867 & -0.28 & 0.71 & -2.93 & 2.80 & 259 & -0.26 & 1.12 & -2.79 & 3.62 \\
\hline Bank & 867 & -0.15 & 0.42 & -1.54 & 1.28 & 259 & -0.22 & 0.47 & -1.39 & 1.73 \\
\hline $\mathrm{AM}$ & 867 & -0.06 & 0.20 & -1.14 & 0.97 & 259 & -0.03 & 0.18 & -0.52 & 0.54 \\
\hline Funds & 867 & 0.01 & 0.07 & -0.28 & 0.24 & 259 & 0.00 & 0.15 & -0.45 & 0.39 \\
\hline Secunities & 867 & -0.07 & 0.43 & -2.01 & 1.88 & 259 & 0.00 & 0.42 & -1.25 & 1.26 \\
\hline Foreign Spot & 802 & 0.49 & 0.76 & -1.57 & 5.62 & 259 & 0.92 & 0.79 & -1.39 & 3.84 \\
\hline DFI Spot & 802 & 0.78 & 1.55 & -5.91 & 7.76 & 259 & 0.51 & 0.78 & -2.05 & 2.44 \\
\hline Bank & 802 & 0.42 & 0.74 & -1.92 & 3.30 & 259 & 0.47 & 0.51 & -1.12 & 1.89 \\
\hline AM & 802 & -0.02 & 0.91 & -3.80 & 4.13 & 259 & 0.02 & 0.27 & -0.93 & 0.83 \\
\hline Funds & 802 & 0.38 & 0.68 & -2.06 & 3.61 & 259 & 0.02 & 0.42 & -1.68 & 1.39 \\
\hline \multirow{3}{*}{ Variable } & \multicolumn{5}{|c|}{ 3-year KTB } & \multicolumn{5}{|c|}{ 10-year KTB } \\
\hline & \multicolumn{5}{|c|}{ Post-crisis } & \multicolumn{5}{|c|}{ Post-crisis } \\
\hline & Obs & Mean & Std. Dev. & Min & Max & Obs & Mean & Std. Dev. & Min & Max \\
\hline Foreign Futures & 749 & 0.06 & 1.08 & -4.40 & 3.84 & 588 & -0.06 & 0.32 & -2.42 & 2.61 \\
\hline DFI Futures & 749 & -0.05 & 1.05 & -3.78 & 4.29 & 588 & 0.08 & 0.34 & -2.38 & 2.74 \\
\hline Bank & 749 & -0.01 & 0.36 & -1.37 & 0.88 & 588 & 0.13 & 0.53 & -2.43 & 3.18 \\
\hline $\mathrm{AM}$ & 749 & -0.02 & 0.06 & -0.22 & 0.21 & 588 & -0.04 & 0.08 & -0.64 & 0.34 \\
\hline Funds & 749 & -0.02 & 0.13 & -0.67 & 0.56 & 588 & 0.02 & 0.28 & -0.89 & 2.08 \\
\hline Secunities & 749 & 0.01 & 0.45 & -1.47 & 1.58 & 588 & -0.04 & 0.77 & -4.12 & 4.77 \\
\hline Foreign Spot & 749 & 0.88 & 0.53 & -2.05 & 4.76 & 619 & 0.45 & 0.62 & -2.14 & 3.72 \\
\hline DFI Spot & 749 & 1.72 & 1.23 & -2.77 & 6.26 & 619 & 2.48 & 2.07 & -4.01 & 14.23 \\
\hline Bank & 749 & 1.16 & 0.71 & -0.71 & 4.16 & 619 & -0.39 & 0.68 & -3.18 & 1.92 \\
\hline $\mathrm{AM}$ & 749 & 0.40 & 0.57 & -1.49 & 2.85 & 619 & 0.33 & 0.29 & -0.40 & 2.14 \\
\hline Funds & 749 & 0.16 & 0.35 & -1.32 & 1.59 & 619 & 2.55 & 1.91 & -1.49 & 16.39 \\
\hline
\end{tabular}

Notes: DFI $=$ domestic financial institutions, $A M=$ asset management

We examine the pattern of unexpected components'14) in relation to the BOK monetary policy stance and Figures 2 presents the unexpected component of net purchases and net long positions of various investor groups in both markets in relation to the BOK monetary policy stance. Overall, the unexpected components of foreign investors' net purchases and positions are negatives in both the cash and futures markets, while those of DFI s are more positives

In the cash market, the unexpected component of foreigner investors' net purchases was more positively responsive to the monetary policy changes in the pre-crisis period, but became more negative through the global crisis and in the post-crisis period right after the crisis (Figure 2. a). On the other hand, the

14) By the nature of the regression model, the unexpected component of net purchasesis averaged zero as they are residuals from $E Q 1$. 
unexpected component of DFIs' net purchases increased in the precrisis period running up to the crisis, but decreased in the crisis and post-crisis period until very recently. We note that for 3-year bond futures, the unexpected component of foreigners' net positions are generally negative in response to the BOK's aggressive policy actions (either increasing or decreasing the base rate), while DFIs tend to take net long positions in the futures markets as the counterparts to the foreigners' positions during those periods (Figure 2. b).

These findings show that foreigners and DFIs take opposite positions in their unexpected net positions in the futures market whenever the BOK takes aggressive policy actions. Furthermore, although foreigners have overall net long positions in the futures market, they tend to take net short positions when unexpectedinformation arrives in the market.

Figure 2: Unexpected Component of Net Purchases and Positions by Foreign and Domestic Finance Institutions
(a. 3-Year Treasury Cash Market)
(b. 3-Year Treasury Futures Market)
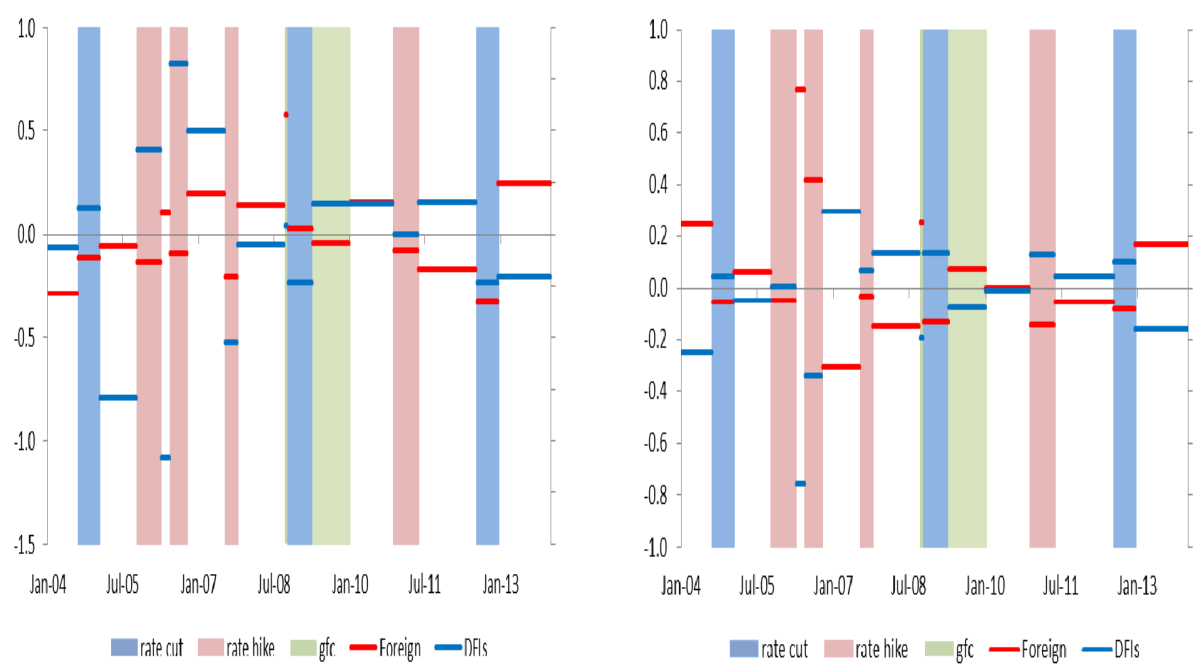

Notes: Futures price index scaled by 100 .

Blue shade $=$ periods of interest rate cuts, red shade $=$ periods of interest hikes, green shade = global financial crisis, DFI = domestic finance institution, gfc = global financial crisis . 


\section{Empirical Specification and Results}

\section{Empirical Specification}

Following the specifications of Brandt, Kavajez, and Underwood (2007) in determining which market (cash or futures) leads to price discovery in Korean Treasury bonds, in which investor groups drive the process and in which component (expected or unexpected) plays a stronger role, we estimate cash (futures) returns on contemporaneous and lagged net purchases (net long positions) by investor groups as well as lagged cross market returns, 15$)$ given by:

$$
\begin{aligned}
R_{t}= & a+\beta_{1} F r F_{t}+\beta_{2} F r F_{t-1}+\beta_{3} F r S_{t}+\beta_{4} F r S_{t-1}+\beta_{5} D F I F_{t} \\
& +\beta_{6} D F I F_{t-1}+\beta_{7} D F I S_{t}+\beta_{8} D F I S_{t-1}+\beta_{9} C R_{t-1}+\varepsilon_{t}
\end{aligned}
$$

where $R$ refers to cash or futures returns, $F r F$ is the foreign net purchases in the futures market, FrSis their net purchases in the cash market, DFIF is the DFI s net purchases in the futures market, DFIS is their net purchases in the cash market, $C R$ is the cross market returns, and $\varepsilon$ is the error term. We then breakdown the net purchases of investor groups by expected and unexpected components derived in Section III . 3. We repeat these two estimations for the three subperiods (pre-crisis, crisis, and post-crisis) and then later disaggregateDFI sinto banks, asset management, funds, and seaurities.

We stress several important points on our model specification. First, we indude both contemporaneous and lagged net purchases to account for the instantaneous as well as delayed impact of trading on returns. In contrast, Brandt, Kavajec, and Underwood (2007) do not indude lagged terms. Second, we indude lagged cross-market returns to confirm whether or not previous trading day returns in the cash market exert significant influence on present day futures returns, and vice versa. Third, we break down the net purchases and net long positions by their

15) The Brandt, Kavajez, and Underwood (2007) specification of the interaction between the Spot and futures market al so looks into different investor categories across maturities. Unlike their spedification, we include lagged terms and cross market returns to determine their contribution to net positions. 
expected and unexpected component, following Richards (2005). Here, we expect that both components exert price effects although the size of that effect could be different depending on how traders extract new information from the trading behaviors. Fourth, unlike other studies such as those from Huang and Zhang (1995), Kim, Szakmary, and Schwarz (1999), and Theissen (2012) - who used returns and price data for error correction models to test price discovery between cash and futures markets - we follow Brandt, Kavajec, and Underwood (2007), who regressed returns on cash and futures net purchases and net long positions in both markets to fully utilize the information content from net transaction data to assess KTB price discovery.16)

Tables 5 to 15 present our estimation results 17$)$. We first present the full sample period (Tables 5, 6, and 7) before showing the pre-crisis (Tables 8 and 9), crisis (Tables 10 and 11) and post-crisis (12 and 13) periods. Tables 7, 9, 11, and 13 present the of expected and unexpected components by sample periods.

We tested whether the net purchases in both cash and futures markets are serially correlated. Using the Ljung-Box test for serial correlation, we were not able to reject the null hypothesis of no serial correlation at one lag. In addition, since we find that the net long positions of foreign investors and DFI s are highly correlated through the variance inflation factor test for multicollinearity, we used the regression residuals of foreign futures on DFI futures as a substitute for DFI futures. 18) Therefore, the results presented in specifications (3) and (6) use the residuals as a substitute for the DFI futures. The model fit for all specifications (as indicated by the R-squared) is not very high, ranging from $8 \%$ to less than $30 \%$ The goodness of fit would improve if we induded contemporaneous cross-market returns, but the effects of these are overwhelmingly large, given that these two markets are likely to be cointegrated, as suggested in the previous literature.

16) Brandt, Kavajecz, and Underwood (2007) cite several studies that argue that net order flows is the mechanism through which information is impounded into prices. These prices are, in turn, used by other authors to test price discovery via an error correction model.

17) We only show analysis results for only 3-year cash and futures KTBs. We have done the same analysis for 10-year KTBs, but the results were overall insignificant. In cases where the results are significant, they were in line with the findings for 3-year. The empirical results for 10-year can be available upon request.

18) We found that foreign and domestic finance institutions futures are highly correlated as they of similar magnitude, but in the opposite direction. We purged the correlation between the two to capture the real trading pattern of both investor groups when used in the same specification. 


\section{Analysis of Results}

Tables 5 to 13 suggest the following findings. First, comparing Tables 5 and 6 , we note that when the model specification considers interaction between the cash and futures market, it has a higher model fit (Table 6) compared to the model specification where we consider own-market net transactions only (Table 5). In fact, specification (3) in Table 5, where we look into the impact of net purchases in the cash market of foreigners and DFIs, the R-squared is only about $6 \%$ But when we consider net purchases and net long positions in both cash and futures markets for foreigners and DFI s in specification (3) of Table 6, the R-squared increases to about $12 \%$ In contrast, in specification (6) of both tables for the futures returns, the difference between the two is quite small (11\% versus 13\%). Drawing on Brandt, Kavajez, and Underwood (2007), this provides evidence that price discovery takes place in the futures market. That is, the futures market contributes significantly more to price movement in the cash market, compared to the cash market contribution to the futures market. Furthermore, the net long positions of foreigners are statistically significant, with higher coefficients than DFIs for the price effect in Table 6, offering evidence that it is foreigners who drive the discovery process in the futures market.

Second, the estimated coefficients for contemporaneous and lagged net purchases and net long positions show opposing signs; that is, when one is positive the other is negative. This could suggest a trading pattern in which investors adjust positions as they reassess information from the previous day. Excess buying the previous day, which suggests lower prices, will increase the price in the current trading period and lead to lower net purchases and net long positions, hence the negative sign.

In Table 6, the foreign net long positions in the futures market exerts significant positive influence on both cash and futures returns. The net futures positions of DFI's are also significant, but their price effects are almost equal, although of opposite sign to those of foreign net futures positions. This implies that DFIs take offsetting positions for the foreign investors' net positions in the futures market. 
Table 5: Full Sample (Own-Market Estimates)

\begin{tabular}{|c|c|c|c|c|c|c|}
\hline \multirow{2}{*}{ VARIABLES } & (1) & (2) & (3) & $(4)$ & $(5)$ & $(6)$ \\
\hline & sreturns3 & sreturns3 & sreturns3 & freturn3 & freturn3 & freturn3 \\
\hline Foreign (Spot/Futures) & $\begin{array}{l}0.00055 \\
(0.00123)\end{array}$ & & $\begin{array}{l}0.00008 \\
(0.00141)\end{array}$ & $\begin{array}{l}0.01631^{* * *} \\
(0.00116)\end{array}$ & & $\begin{array}{l}0.01887^{* * *} \\
(0.00118)\end{array}$ \\
\hline Lag Foreign (Spot/Futures) & $\begin{array}{l}-0.00190^{*} \\
(0.00105)\end{array}$ & & $\begin{array}{l}-0.00147 \\
(0.00123)\end{array}$ & $\begin{array}{l}-0.00298^{* * *} \\
(0.00109)\end{array}$ & & $\begin{array}{l}-0.00411^{* * * *} \\
(0.00117)\end{array}$ \\
\hline DFls (Spot/Futures) & & $\begin{array}{l}-0.00604^{* * *} \\
(0.00061)\end{array}$ & $\begin{array}{l}-0.00602^{* * *} \\
(0.00061)\end{array}$ & & $\begin{array}{l}-0.01607^{\text {**** }} \\
(0.00119)\end{array}$ & $\begin{array}{l}0.00654^{* * *} \\
(0.00172)\end{array}$ \\
\hline Lag DFls (Spot/Futures) & & $\begin{array}{l}0.00064 \\
(0.00055)\end{array}$ & $\begin{array}{l}0.00061 \\
(0.00055)\end{array}$ & & $\begin{array}{l}0.00306^{* * *} \\
(0.00109)\end{array}$ & $\begin{array}{l}-0.00252^{*} \\
(0.00143)\end{array}$ \\
\hline Lag Cross Market Returns & $\begin{array}{l}0.02123 \\
(0.02730)\end{array}$ & $\begin{array}{l}0.00786 \\
(0.02790)\end{array}$ & $\begin{array}{l}0.00795 \\
(0.02797)\end{array}$ & $\begin{array}{l}-0.00117 \\
(0.03485)\end{array}$ & $\begin{array}{l}0.00400 \\
(0.03490)\end{array}$ & $\begin{array}{l}0.00599 \\
(0.03502)\end{array}$ \\
\hline Constant & $\begin{array}{l}0.00240 \\
(0.00341)\end{array}$ & $\begin{array}{l}0.00789^{* *} \\
(0.00332)\end{array}$ & $\begin{array}{l}0.00888^{* *} \\
(0.00349)\end{array}$ & $\begin{array}{l}0.00444 \\
(0.00343)\end{array}$ & $\begin{array}{l}0.00443 \\
(0.00343)\end{array}$ & $\begin{array}{l}0.00452 \\
(0.00341)\end{array}$ \\
\hline Observations & 2,067 & 2,067 & 2,067 & 2,066 & 2,066 & 2,066 \\
\hline R-squared & 0.00176 & 0.05601 & 0.05675 & 0.10067 & 0.09656 & 0.10998 \\
\hline
\end{tabular}

Notes: Specifications (1) to (3) refers to spot net purchases. Specifications (4) to (6) futures net long positions. Robust standard errors in parentheses; *** $p<0.01$, ** $p<0.05, \quad$ * $p<0.1$

Table 6: Full Sample (Market Interaction Estimates)

\begin{tabular}{|c|c|c|c|c|c|c|}
\hline \multirow{2}{*}{ VARIABLES } & $(1)$ & (2) & (3) & (4) & (5) & (6) \\
\hline & sreturns3 & sreturns3 & sreturns3 & freturn3 & freturn3 & freturn3 \\
\hline \multirow[t]{2}{*}{ Futures Foreign } & $0.01359^{* * *}$ & & $0.01161^{* * *}$ & $0.01629^{* * *}$ & & $0.01424^{* * *}$ \\
\hline & $(0.00105)$ & & $(0.00110)$ & $(0.00117)$ & & $(0.00124)$ \\
\hline \multirow[t]{2}{*}{ Lag Futures Foreign } & $-0.00191 *$ & & $-0.00185^{*}$ & $-0.00304^{* * *}$ & & $-0.00296 * * *$ \\
\hline & $(0.00098)$ & & $(0.00102)$ & $(0.00109)$ & & $(0.00112)$ \\
\hline \multirow[t]{2}{*}{ Spot Foreign } & 0.00080 & & 0.00037 & 0.00093 & & 0.00048 \\
\hline & $(0.00115)$ & & $(0.00131)$ & $(0.00132)$ & & $(0.00147)$ \\
\hline \multirow[t]{2}{*}{ Lag Spot Foreign } & -0.00161 & & -0.00135 & -0.00169 & & -0.00140 \\
\hline & $(0.00101)$ & & $(0.00114)$ & $(0.00113)$ & & $(0.00126)$ \\
\hline \multirow[t]{2}{*}{ Futures DFls } & & $-0.01157^{* * *}$ & 0.00385 & & $-0.01417^{* * *}$ & 0.00290 \\
\hline & & $(0.00109)$ & $(0.00590)$ & & $(0.00122)$ & $(0.00647)$ \\
\hline \multirow[t]{2}{*}{ Lag Futures DFls } & & $0.00178^{*}$ & 0.00607 & & $0.00287^{* *}$ & 0.00810 \\
\hline & & $(0.00101)$ & $(0.00498)$ & & $(0.00112)$ & $(0.00541)$ \\
\hline \multirow[t]{2}{*}{ Spot DFls } & & $-0.00460^{* * *}$ & $-0.00457^{* * *}$ & & $-0.00490^{* * *}$ & $-0.00487^{* * *}$ \\
\hline & & $(0.00059)$ & $(0.00059)$ & & $(0.00066)$ & $(0.00065)$ \\
\hline \multirow[t]{2}{*}{ Lag Spot DFls } & & $0.00094^{*}$ & $0.00093^{*}$ & & $0.00123^{* *}$ & $0.00122^{* *}$ \\
\hline & & $(0.00054)$ & $(0.00054)$ & & $(0.00062)$ & $(0.00061)$ \\
\hline \multirow[t]{2}{*}{ Lag Cross Market Returns } & 0.01718 & 0.01498 & 0.01009 & -0.00031 & -0.00063 & -0.00687 \\
\hline & $(0.02843)$ & $(0.02846)$ & $(0.02846)$ & $(0.03488)$ & $(0.03531)$ & $(0.03536)$ \\
\hline \multirow[t]{2}{*}{ Constant } & 0.00072 & 0.00467 & 0.00556 & 0.00497 & $0.00895^{* *}$ & $0.00980^{* *}$ \\
\hline & $(0.00328)$ & $(0.00327)$ & $(0.00345)$ & $(0.00361)$ & $(0.00364)$ & $(0.00382)$ \\
\hline Observations & 2,067 & 2,067 & 2,067 & 2,066 & 2,066 & 2,066 \\
\hline R-squared & 0.08978 & 0.11540 & 0.12013 & 0.10154 & 0.12467 & 0.12933 \\
\hline
\end{tabular}

Notes: Robust standard errors in parentheses; *** $p<0.01$, ** $p<0.05$, * $p<0.1$ 
On the other hand, foreign trading in the cash market does not seem to influence the prices in either market, whereas DFIs' net purchases in the cash market have a significant, negative effect on cash and futures returns.

Third, in Table 7, for both foreign net long positions in the futures market and DFIs' net purchases in the cash market, expected components appear to exert more price influence, given the magnitude of the coefficients with statistical significance. Similar to the results of Richards (2005), our estimates show that both expected and unexpected components play significant roles in bond pricing. However, careful comparison between them in specification (3) suggests that for foreign futures trading, the effect of the expected component is just a bit higher than the unexpected ( 0.026 versus 0.011 in the contemporary returns, and 0.013 versus 0.007 in the sum of contemporary and previous day returns). For DFI cash trading, the effect of the unexpected component is much smaller, while these effects are weaker for both expected and unexpected components (-0.02 vs -0.004 ; -0.008 vs. -0.001$)$.

This can be interpreted as an indication of how foreign investors' net futures positions change unexpectedly will carry valuable additional information. That is, the market extracts extra information, be it their private information or idiosyncratic portfolio rebalancing, from their trading behaviors. On the other hand, DFI transactions in the 3-year cash market are more predictable provided that their assessment of overall economic conditions is expected. This finding holds true for futures returns as well.

Fourth, compared to Table 6, the subperiod estimates show several noteworthy issues. In the pre-crisis period (Table 8), the effects of lagged transaction variables are insignificant, while the effects of lagged cross-market returns are significant. The effects of lagged, cross-market returns disappear in crisis and post-crisis periods (Tables 10 and 12). The results seem to suggest that in the pre-crisis period, investors did not pay as much attention as the later periods to the information content of trading. Rather, they tried to extract information from observing prices in other markets and exploited arbitrage opportunities. Over time, investors have become much more active in their information gathering from intraday price movements. As information transmission quickens to nearly instant, 
the effects of lagged returns are not important anymore. This reasoning seems to have worked well in recent years, whereby cash and futures trading has been much more dosely watched and there is now instant and intraday price transmission between the two markets.

In the crisis period (Table 10), the effects of foreign net positions in the futures market are significant and large compared to pre and post-crisis periods. However, during 2008-2009, the share of foreign net long positions in 3-year KTB futures was not very big in absolute terms, although compared to DFIs, their transactions were relatively larger. In the post-crisis period (Table 12), the effect of the DFIs' net long positions in the futures market is greater than the effect of foreign investors' net long positions. The price effect of DFI trading increased significantly after the crisis, reflecting their increased size and sophistication.

In terms of the expected and unexpected components subperiods, Tables 9, 11, and 13 show that the unexpected foreign net long positions in the futures market seem to consistently exert price influence across subperiods. However, our results also confirm the significance of the expected component, with its larger price effect, but the results are not consistent across periods and specifications. The greater impact of the expected component, albeit inconsistent across specifications and periods, might be due to increased transparency and access to information has raised the effectiveness of expected net long positions, and therefore increased their effect on returns relative to unexpected net long positions.

What these findings imply is that, over the sample period, the trading patterns of investor groups have evolved. The pricing effect of foreign participation is consistently significant and positive on both 3-year cash and futures markets across all subperiods and particularly high during the crisis period. During the crisis period, both the expected and unexpected components of foreign transactions exert strong influence on prices, but the impact of the expected component is substantially higher than that of the unexpected, and visibly increases compared to pre and post-crisis periods. This suggests very active foreign trading in response to announcements in global and domestic macroeconomic conditions. On the other hand, foreign participation in the cash market has a negligible, if any, price effect. 
Table 7: Full Sample (Expected and Unexpected Component Estimates)

\begin{tabular}{|c|c|c|c|c|c|c|}
\hline \multirow{2}{*}{ VARIABLES } & (1) & (2) & (3) & (4) & (5) & (6) \\
\hline & sreturns3 & sreturns3 & sreturns3 & freturn3 & freturn3 & freturn3 \\
\hline \multirow[t]{2}{*}{ Unexpected Futures Foreign } & $0.01273^{* * *}$ & & $0.01119^{* * *}$ & $0.01561^{* * *}$ & & $0.01401^{* * *}$ \\
\hline & $(0.00117)$ & & $(0.00124)$ & $(0.00132)$ & & $(0.00141)$ \\
\hline \multirow[t]{2}{*}{ Lag Unexpected Futures Foreign } & $-0.00357^{* * *}$ & & $-0.00448^{* * *}$ & $-0.00497^{* * *}$ & & $-0.00612^{* * *}$ \\
\hline & $(0.00137)$ & & $(0.00140)$ & $(0.00152)$ & & $(0.00153)$ \\
\hline \multirow[t]{2}{*}{ Expected Futures Foreign } & $0.02458^{* * *}$ & & $0.02602^{* * *}$ & $0.02823^{* * *}$ & & $0.03059^{* * *}$ \\
\hline & $(0.00603)$ & & $(0.00624)$ & $(0.00678)$ & & $(0.00697)$ \\
\hline \multirow[t]{2}{*}{ Lag Expected Futures Foreign } & $-0.01130^{* *}$ & & $-0.01325^{* *}$ & $-0.01310^{* *}$ & & $-0.01516^{* *}$ \\
\hline & $(0.00571)$ & & $(0.00566)$ & $(0.00634)$ & & $(0.00626)$ \\
\hline \multirow[t]{2}{*}{ Unexpected Spot Foreign } & 0.00068 & & 0.00010 & 0.00099 & & 0.00040 \\
\hline & $(0.00149)$ & & $(0.00166)$ & $(0.00170)$ & & $(0.00187)$ \\
\hline \multirow[t]{2}{*}{ Lag Unexpected Spot Foreign } & -0.00113 & & -0.00075 & -0.00174 & & -0.00141 \\
\hline & $(0.00151)$ & & $(0.00161)$ & $(0.00172)$ & & $(0.00183)$ \\
\hline \multirow[t]{2}{*}{ Expected Spot Foreign } & 0.00595 & & 0.00653 & 0.00982 & & 0.01102 \\
\hline & $(0.00823)$ & & $(0.00808)$ & $(0.00935)$ & & $(0.00914)$ \\
\hline \multirow[t]{2}{*}{ Lag Expected Spot Foreign } & -0.00437 & & -0.00598 & -0.00584 & & -0.00753 \\
\hline & $(0.00834)$ & & $(0.00824)$ & $(0.00951)$ & & $(0.00941)$ \\
\hline \multirow[t]{2}{*}{ Unexpected Futures DFIs } & & $-0.01109^{* * *}$ & -0.00117 & & $-0.01384^{* * *}$ & -0.00225 \\
\hline & & $(0.00123)$ & $(0.00630)$ & & $(0.00139)$ & $(0.00710)$ \\
\hline \multirow[t]{2}{*}{ Lag Unexpected Futures DFls } & & $0.00434^{* * *}$ & $0.01970^{* * *}$ & & $0.00594^{* * *}$ & $0.02533^{* * *}$ \\
\hline & & $(0.00142)$ & $(0.00630)$ & & $(0.00155)$ & $(0.00700)$ \\
\hline \multirow[t]{2}{*}{ Expected Futures DFls } & & $-0.02739^{* * *}$ & $-0.07883^{* *}$ & & $-0.03252^{* * *}$ & $-0.11144^{* * *}$ \\
\hline & & $(0.00646)$ & $(0.03212)$ & & $(0.00714)$ & $(0.03501)$ \\
\hline \multirow[t]{2}{*}{ Lag Expected Futures DFls } & & $0.01456^{* *}$ & 0.03654 & & $0.01716^{* * *}$ & 0.05195 \\
\hline & & $(0.00589)$ & $(0.03164)$ & & $(0.00646)$ & $(0.03556)$ \\
\hline \multirow[t]{2}{*}{ Unexpected Spot DFls } & & $-0.00409^{* * *}$ & $-0.00415^{* * *}$ & & $-0.00437^{* * *}$ & $-0.00443^{* * *}$ \\
\hline & & $(0.00066)$ & $(0.00065)$ & & $(0.00074)$ & $(0.00073)$ \\
\hline \multirow[t]{2}{*}{ Lag Unexpected Spot DFls } & & $0.00258^{* * *}$ & $0.00272^{* * *}$ & & $0.00303^{* * *}$ & $0.00322^{* * *}$ \\
\hline & & $(0.00069)$ & $(0.00070)$ & & $(0.00078)$ & $(0.00078)$ \\
\hline \multirow[t]{2}{*}{ Expected Spot DFls } & & $-0.02094^{* * *}$ & $-0.02109^{* * *}$ & & $-0.02269^{* * *}$ & $-0.02292^{* * *}$ \\
\hline & & $(0.00497)$ & $(0.00495)$ & & $(0.00556)$ & $(0.00553)$ \\
\hline \multirow[t]{2}{*}{ Lag Expected Spot DFls } & & $0.01279^{* * *}$ & $0.01303^{* * *}$ & & $0.01434^{* * *}$ & $0.01487^{* * *}$ \\
\hline & & $(0.00425)$ & $(0.00428)$ & & $(0.00473)$ & $(0.00475)$ \\
\hline \multirow[t]{2}{*}{ Lag Cross Market Returns } & 0.01273 & -0.01316 & -0.00203 & -0.00199 & -0.03128 & -0.01293 \\
\hline & $(0.03103)$ & $(0.03495)$ & $(0.03482)$ & $(0.03780)$ & $(0.04312)$ & $(0.04307)$ \\
\hline \multirow[t]{2}{*}{ Constant } & 0.00052 & $0.01102^{*}$ & 0.01034 & 0.00329 & $0.01549^{* *}$ & 0.01242 \\
\hline & $(0.00581)$ & $(0.00575)$ & $(0.00788)$ & $(0.00638)$ & $(0.00641)$ & $(0.00860)$ \\
\hline Observations & 1,645 & 1,645 & 1,645 & 1,645 & 1,645 & 1,645 \\
\hline R-squared & 0.08933 & 0.12707 & 0.13326 & 0.10351 & 0.13760 & 0.14610 \\
\hline
\end{tabular}

Notes: Robust standard errors in parentheses; *** $p<0.01, * * p<0.05, \quad * p<0.1$ 
Table 8: Pre-Crisis Estimates

\begin{tabular}{|c|c|c|c|c|c|c|}
\hline \multirow{2}{*}{ VARIABLES } & (1) & (2) & (3) & (4) & (5) & (6) \\
\hline & sreturns3 & sreturns3 & sreturns3 & freturn3 & freturn3 & freturn3 \\
\hline \multirow[t]{2}{*}{ Futures Foreign } & $0.01124^{* * *}$ & & $0.01075^{* * *}$ & $0.01317^{* * *}$ & & $0.01270^{* * *}$ \\
\hline & $(0.00119)$ & & $(0.00121)$ & $(0.00130)$ & & $(0.00133)$ \\
\hline \multirow[t]{2}{*}{ Lag Futures Foreign } & -0.00081 & & -0.00081 & -0.00144 & & -0.00150 \\
\hline & $(0.00127)$ & & $(0.00130)$ & $(0.00142)$ & & $(0.00145)$ \\
\hline \multirow[t]{2}{*}{ Spot Foreign } & -0.00161 & & -0.00251 & -0.00194 & & -0.00288 \\
\hline & $(0.00187)$ & & $(0.00198)$ & $(0.00209)$ & & $(0.00220)$ \\
\hline \multirow[t]{2}{*}{ Lag Spot Foreign } & -0.00231 & & -0.00180 & -0.00262 & & -0.00204 \\
\hline & $(0.00167)$ & & $(0.00190)$ & $(0.00197)$ & & $(0.00221)$ \\
\hline \multirow[t]{2}{*}{ Futures DFls } & & $-0.01070^{* * *}$ & -0.00177 & & $-0.01265^{* * *}$ & -0.00332 \\
\hline & & $(0.00120)$ & (0.00598) & & $(0.00131)$ & $(0.00662)$ \\
\hline \multirow[t]{2}{*}{ Lag Futures DFls } & & 0.00092 & 0.00549 & & 0.00164 & $0.00844^{*}$ \\
\hline & & $(0.00128)$ & $(0.00426)$ & & $(0.00143)$ & $(0.00492)$ \\
\hline \multirow[t]{2}{*}{ Spot DFls } & & $-0.00233^{* * *}$ & $-0.00253^{* * *}$ & & $-0.00244^{* * *}$ & $-0.00269^{* * *}$ \\
\hline & & $(0.00067)$ & $(0.00067)$ & & $(0.00074)$ & $(0.00075)$ \\
\hline \multirow[t]{2}{*}{ Lag Spot DFls } & & 0.00088 & 0.00075 & & 0.00112 & 0.00097 \\
\hline & & $(0.00069)$ & $(0.00069)$ & & $(0.00079)$ & $(0.00079)$ \\
\hline \multirow[t]{2}{*}{ Lag Oross Merket Returns } & $0.10453^{* * *}$ & $0.10265^{* * *}$ & $0.09440^{* *}$ & $0.11010^{* *}$ & $0.11121^{* *}$ & $0.09996^{* *}$ \\
\hline & $(0.03733)$ & (0.03708) & $(0.03697)$ & $(0.04669)$ & $(0.04673)$ & $(0.04648)$ \\
\hline \multirow[t]{2}{*}{ Constant } & -0.00141 & -0.00191 & 0.00036 & 0.00057 & -0.00034 & 0.00225 \\
\hline & $(0.00433)$ & $(0.00424)$ & $(0.00438)$ & $(0.00483)$ & $(0.00474)$ & $(0.00489)$ \\
\hline Observations & 920 & 920 & 920 & 919 & 919 & 919 \\
\hline R-squared & 0.11615 & 0.12214 & 0.12932 & 0.11985 & 0.12501 & 0.13263 \\
\hline
\end{tabular}

Notes: Robust standard errors in parentheses; $* * * p<0.01, * * p<0.05$, * $p<0.1$

\section{Table 9: Pre-Crisis Estimates (Expected and Unexpected)}

\begin{tabular}{|c|c|c|c|c|c|c|}
\hline \multirow{2}{*}{ VARIABLES } & $(1)$ & $(2)$ & $(3)$ & $(4)$ & $(5)$ & (6) \\
\hline & sreturns3 & sreturns3 & sreturns3 & freturn3 & freturn3 & freturn3 \\
\hline \multirow[t]{2}{*}{ Unexpected Futures Foreign } & $0.0102^{* * *}$ & & $0.0101^{* * *}$ & $0.0123^{* * *}$ & & $0.0122^{* * *}$ \\
\hline & (0.00122) & & $(0.00127)$ & $(0.00137)$ & & $(0.00143)$ \\
\hline \multirow[t]{2}{*}{ Lag Unexpected Futures Foreign } & -0.00191 & & $-0.00241^{*}$ & $-0.00263^{*}$ & & $-0.00330^{* *}$ \\
\hline & $(0.00133)$ & & $(0.00137)$ & $(0.00151)$ & & $(0.00154)$ \\
\hline \multirow[t]{2}{*}{ Expected Futures Foreign } & 0.0173 & & $0.0199^{*}$ & 0.0182 & & 0.0211 \\
\hline & $(0.0107)$ & & $(0.0115)$ & $(0.0124)$ & & $(0.0135)$ \\
\hline \multirow[t]{2}{*}{ Lag Expected Futures Foreign } & -0.00417 & & -0.00173 & -0.00380 & & -0.00130 \\
\hline & $(0.00975)$ & & $(0.00880)$ & $(0.0112)$ & & $(0.0101)$ \\
\hline \multirow[t]{2}{*}{ Unexpected Spot Foreign } & -0.00273 & & -0.00337 & -0.00289 & & -0.00361 \\
\hline & $(0.00209)$ & & $(0.00217)$ & $(0.00237)$ & & $(0.00247)$ \\
\hline \multirow[t]{2}{*}{ Lag Unexpected Spot Foreign } & $-0.00413^{*}$ & & -0.00354 & -0.00435 & & -0.00378 \\
\hline & $(0.00229)$ & & $(0.00244)$ & $(0.00267)$ & & $(0.00283)$ \\
\hline \multirow[t]{2}{*}{ Expected Spot Foreign } & 0.0179 & & 0.0171 & 0.0197 & & 0.0193 \\
\hline & $(0.0119)$ & & $(0.0118)$ & $(0.0139)$ & & $(0.0138)$ \\
\hline \multirow[t]{2}{*}{ Lag Expected Spot Foreign } & -0.0187 & & -0.0187 & -0.0215 & & -0.0212 \\
\hline & $(0.0132)$ & & $(0.0133)$ & $(0.0157)$ & & $(0.0158)$ \\
\hline Unexpected Futures DFls & & $\begin{array}{l}-0.0101^{* * *} \\
(0.00129)\end{array}$ & -0.00837 & & $-0.0121^{* * *}$ & -0.00954 \\
\hline
\end{tabular}




\section{$29 \quad$ BOK Working Paper No.2015-8 (2015.3)}

(Table 9, continued)

\begin{tabular}{|c|c|c|c|c|c|c|}
\hline \multirow{2}{*}{ VARIABLES } & (1) & (2) & (3) & (4) & (5) & (6) \\
\hline & sreturns3 & sreturns3 & sreturns3 & freturn3 & freturn3 & freturn3 \\
\hline \multirow[t]{2}{*}{ Lag Unexpected Futures DFls } & & $0.00229^{*}$ & $0.0110^{*}$ & & $0.00313^{* *}$ & $0.0151^{* *}$ \\
\hline & & $(0.00133)$ & $(0.00581)$ & & $(0.00150)$ & $(0.00671)$ \\
\hline \multirow[t]{2}{*}{ Expected Futures DFls } & & $-0.0222^{*}$ & -0.0386 & & $-0.0236^{*}$ & -0.0476 \\
\hline & & $(0.0122)$ & $(0.0430)$ & & $(0.0143)$ & $(0.0485)$ \\
\hline \multirow[t]{2}{*}{ Lag Expected Futures DFls } & & 0.00299 & 0.000503 & & 0.00298 & 0.00478 \\
\hline & & $(0.00909)$ & $(0.0394)$ & & $(0.0104)$ & $(0.0446)$ \\
\hline \multirow[t]{2}{*}{ Unexpected Spot DFIs } & & $-0.00210^{* * *}$ & $-0.00235^{* * *}$ & & $-0.00230^{* * *}$ & $-0.00260^{* * *}$ \\
\hline & & $(0.000729)$ & $(0.000714)$ & & $(0.000827)$ & $(0.000814)$ \\
\hline \multirow[t]{2}{*}{ Lag Unexpected Spot DFls } & & 0.00167 & 0.00163 & & 0.00174 & 0.00174 \\
\hline & & $(0.00106)$ & $(0.00104)$ & & $(0.00123)$ & $(0.00121)$ \\
\hline \multirow[t]{2}{*}{ Expected Spot DFls } & & -0.00968 & -0.00962 & & -0.00863 & -0.00894 \\
\hline & & $(0.00756)$ & $(0.00696)$ & & $(0.00894)$ & $(0.00810)$ \\
\hline \multirow[t]{2}{*}{ Lag Expected Spot DFls } & & 0.00787 & 0.00713 & & 0.00775 & 0.00718 \\
\hline & & $(0.00606)$ & $(0.00584)$ & & $(0.00693)$ & $(0.00660)$ \\
\hline \multirow[t]{2}{*}{ Lag Cross Market Returns } & $0.0954^{* *}$ & 0.0596 & 0.0612 & $0.103^{*}$ & 0.0715 & 0.0723 \\
\hline & $(0.0437)$ & $(0.0587)$ & $(0.0546)$ & $(0.0565)$ & $(0.0821)$ & $(0.0746)$ \\
\hline \multirow[t]{2}{*}{ Constant } & -0.00322 & -0.00364 & -0.00238 & -0.00152 & -0.00325 & -0.00165 \\
\hline & $(0.00585)$ & $(0.00605)$ & $(0.00770)$ & $(0.00654)$ & $(0.00699)$ & $(0.00879)$ \\
\hline Observations & 726 & 726 & 726 & 726 & 726 & 726 \\
\hline R-squared & 0.118 & 0.126 & 0.137 & 0.123 & 0.129 & 0.141 \\
\hline
\end{tabular}

Notes: Robust standard errors in parentheses; ${ }^{* *} p<0.01, * * p<0.05, \quad{ }^{*} p<0.1$

Table 10: Crisis Estimates

\begin{tabular}{|c|c|c|c|c|c|c|}
\hline \multirow{2}{*}{ VARIABLES } & (1) & (2) & (3) & (4) & (5) & (6) \\
\hline & sreturns3 & sreturns3 & sreturns3 & freturn3 & freturn3 & freturn3 \\
\hline Futures Foreign & $\begin{array}{l}0.03362^{* * *} \\
(0.00551)\end{array}$ & & $\begin{array}{l}0.03032^{* * *} \\
(0.00584)\end{array}$ & $\begin{array}{l}0.04173^{* * *} \\
(0.00607)\end{array}$ & & $\begin{array}{l}0.03875^{* * *} \\
(0.00651)\end{array}$ \\
\hline Lag Futures Foreign & $\begin{array}{l}-0.01326^{* * *} \\
(0.00423)\end{array}$ & & $\begin{array}{l}-0.01378^{* * *} \\
(0.00459)\end{array}$ & $\begin{array}{l}-0.01838^{* * *} \\
(0.00462)\end{array}$ & & $\begin{array}{l}-0.01867^{* * *} \\
(0.00487)\end{array}$ \\
\hline Spot Foreign & $\begin{array}{l}0.00766 \\
(0.00482)\end{array}$ & & $\begin{array}{l}0.00053 \\
(0.00602)\end{array}$ & $\begin{array}{l}0.00608 \\
(0.00543)\end{array}$ & & $\begin{array}{l}-0.00078 \\
(0.00671)\end{array}$ \\
\hline Lag Spot Foreign & $\begin{array}{l}-0.00019 \\
(0.00559)\end{array}$ & & $\begin{array}{l}0.00012 \\
(0.00590)\end{array}$ & $\begin{array}{r}-0.00068 \\
(0.00587)\end{array}$ & & $\begin{array}{l}-0.00018 \\
(0.00622)\end{array}$ \\
\hline Futures DFls & & $\begin{array}{l}-0.02922^{* * *} \\
(0.00539)\end{array}$ & $\begin{array}{l}-0.00483 \\
(0.02489)\end{array}$ & & $\begin{array}{l}-0.03754^{* * *} \\
(0.00604)\end{array}$ & $\begin{array}{l}-0.00944 \\
(0.02658)\end{array}$ \\
\hline Lag Futures DFls & & $\begin{array}{l}0.01349^{* * *} \\
(0.00434)\end{array}$ & $\begin{array}{l}-0.01423 \\
(0.03031)\end{array}$ & & $\begin{array}{l}0.01819^{* * *} \\
(0.00459)\end{array}$ & $\begin{array}{l}-0.01585 \\
(0.03000)\end{array}$ \\
\hline Spot DFls & & $\begin{array}{l}-0.01385^{* * *} \\
(0.00382)\end{array}$ & $\begin{array}{l}-0.01355^{* * *} \\
(0.00425)\end{array}$ & & $\begin{array}{l}-0.01338^{* * *} \\
(0.00415)\end{array}$ & $\begin{array}{l}-0.01328^{* * *} \\
(0.00460)\end{array}$ \\
\hline Lag Spot DFls & & $\begin{array}{l}-0.00257 \\
(0.00334)\end{array}$ & $\begin{array}{l}-0.00287 \\
(0.00350)\end{array}$ & & $\begin{array}{l}-0.00220 \\
(0.00349)\end{array}$ & $\begin{array}{l}-0.00258 \\
(0.00368)\end{array}$ \\
\hline $\begin{array}{l}\text { Lag Cross Market } \\
\text { Returns }\end{array}$ & -0.04208 & -0.04061 & -0.04761 & -0.08516 & -0.08468 & -0.09363 \\
\hline Constant & $\begin{array}{l}(0.06496) \\
-0.00525 \\
(0.01640)\end{array}$ & $\begin{array}{l}(0.06670) \\
0.01036 \\
(0.01357)\end{array}$ & $\begin{array}{l}(0.06694) \\
0.00954 \\
(0.01797)\end{array}$ & $\begin{array}{l}(0.07446) \\
0.00746 \\
(0.01739)\end{array}$ & $\begin{array}{l}(0.07629) \\
0.02050 \\
(0.01468)\end{array}$ & $\begin{array}{l}(0.07647) \\
0.02108 \\
(0.01952)\end{array}$ \\
\hline Observations & 288 & 288 & 288 & 288 & 288 & 288 \\
\hline R-squared & 0.14829 & 0.19642 & 0.20214 & 0.18954 & 0.22507 & 0.23176 \\
\hline
\end{tabular}

Notes: Robust standard errors in parentheses; $* * * p<0.01,{ }^{* *} p<0.05$, ${ }^{*} p<0.1$ 
Table 11: Crisis Estimates (Expected and Unexpected)

\begin{tabular}{|c|c|c|c|c|c|c|}
\hline \multirow{2}{*}{ VARIABLES } & (1) & (2) & (3) & (4) & (5) & (6) \\
\hline & sreturns3 & sreturns3 & sreturns3 & freturn3 & freturn3 & freturn3 \\
\hline \multirow[t]{2}{*}{ Unexpected Futures Foreign } & $0.03024^{* * *}$ & & $0.02670^{* * *}$ & $0.03984^{* * *}$ & & $0.03642^{* * *}$ \\
\hline & $(0.00663)$ & & $(0.00690)$ & $(0.00732)$ & & $(0.00770)$ \\
\hline \multirow[t]{2}{*}{ Lag Unexpected Futures Foreign } & $-0.02577^{* * *}$ & & $-0.01894^{*}$ & $-0.03375^{* * *}$ & & $-0.02679^{* *}$ \\
\hline & $(0.00902)$ & & $(0.00998)$ & $(0.00953)$ & & $(0.01091)$ \\
\hline \multirow[t]{2}{*}{ Expected Futures Foreign } & $0.09851^{* * *}$ & & $0.06878^{* *}$ & $0.11288^{* * *}$ & & $0.08663^{* * *}$ \\
\hline & $(0.02835)$ & & $(0.02902)$ & $(0.02976)$ & & $(0.03125)$ \\
\hline \multirow[t]{2}{*}{ Lag Expected Futures Foreign } & $-0.05696^{* *}$ & & $-0.04478^{*}$ & $-0.06612^{* *}$ & & $-0.05555^{* *}$ \\
\hline & $(0.02575)$ & & $(0.02487)$ & $(0.02741)$ & & $(0.02733)$ \\
\hline \multirow[t]{2}{*}{ Unexpected Spot Foreign } & 0.00911 & & 0.00156 & 0.00725 & & -0.00025 \\
\hline & $(0.00559)$ & & $(0.00685)$ & $(0.00645)$ & & $(0.00770)$ \\
\hline \multirow[t]{2}{*}{ Lag Unexpected Spot Foreign } & 0.01387 & & 0.01153 & 0.01548 & & 0.01347 \\
\hline & $(0.00956)$ & & $(0.01039)$ & $(0.01009)$ & & $(0.01118)$ \\
\hline \multirow[t]{2}{*}{ Expected Spot Foreign } & -0.04301 & & -0.04099 & -0.05183 & & -0.05093 \\
\hline & $(0.03440)$ & & $(0.03297)$ & $(0.03580)$ & & $(0.03549)$ \\
\hline \multirow[t]{2}{*}{ Lag Expected Spot Foreign } & 0.02041 & & 0.02355 & 0.02705 & & 0.03091 \\
\hline & $(0.02880)$ & & $(0.02868)$ & $(0.03062)$ & & $(0.03130)$ \\
\hline \multirow[t]{2}{*}{ Unexpected Futures DFls } & & $-0.02548^{* * *}$ & 0.00754 & & $-0.03464^{* * *}$ & 0.00591 \\
\hline & & $(0.00633)$ & $(0.02676)$ & & $(0.00709)$ & $(0.03012)$ \\
\hline \multirow[t]{2}{*}{ Lag Unexpected Futures DFls } & & $0.02208^{* * *}$ & 0.02886 & & $0.02942^{* * *}$ & 0.02629 \\
\hline & & $(0.00779)$ & $(0.03301)$ & & $(0.00800)$ & $(0.03416)$ \\
\hline \multirow[t]{2}{*}{ Expected Futures DFls } & & $-0.07663^{* * *}$ & -0.09797 & & $-0.09408^{* * *}$ & -0.10225 \\
\hline & & $(0.02478)$ & $(0.09078)$ & & $(0.02600)$ & $(0.09849)$ \\
\hline \multirow[t]{2}{*}{ Lag Expected Futures DFls } & & $0.05404^{* *}$ & 0.04283 & & $0.06496^{* * *}$ & 0.04044 \\
\hline & & $(0.02276)$ & $(0.07993)$ & & $(0.02458)$ & $(0.08772)$ \\
\hline \multirow[t]{2}{*}{ Unexpected Spot DFls } & & $-0.01125^{* * *}$ & $-0.01064^{* *}$ & & $-0.01102^{* *}$ & $-0.01067^{*}$ \\
\hline & & $(0.00430)$ & $(0.00499)$ & & $(0.00478)$ & $(0.00549)$ \\
\hline \multirow[t]{2}{*}{ Lag Unexpected Spot DFls } & & 0.00291 & 0.00327 & & 0.00296 & 0.00318 \\
\hline & & $(0.00312)$ & $(0.00340)$ & & $(0.00344)$ & $(0.00379)$ \\
\hline \multirow[t]{2}{*}{ Expected Spot DFls } & & $-0.06336^{* * *}$ & $-0.06481^{* * *}$ & & $-0.05699^{* * *}$ & $-0.05800^{* *}$ \\
\hline & & $(0.02092)$ & $(0.02215)$ & & $(0.02131)$ & $(0.02286)$ \\
\hline \multirow[t]{2}{*}{ Lag Expected Spot DFls } & & -0.00267 & 0.00197 & & 0.00303 & 0.00794 \\
\hline & & $(0.01820)$ & $(0.01990)$ & & $(0.01984)$ & $(0.02159)$ \\
\hline \multirow[t]{2}{*}{ Lag Cross Market Returns } & $-0.15983^{*}$ & -0.04137 & -0.07315 & $-0.22654^{* *}$ & -0.08679 & -0.13908 \\
\hline & $(0.08399)$ & $(0.08151)$ & $(0.09352)$ & $(0.08963)$ & $(0.08807)$ & $(0.09901)$ \\
\hline \multirow[t]{2}{*}{ Constant } & 0.02272 & $0.03643^{*}$ & 0.05034 & 0.03431 & $0.04066^{*}$ & 0.05624 \\
\hline & $(0.03578)$ & $(0.02198)$ & $(0.03857)$ & $(0.03655)$ & $(0.02390)$ & $(0.04124)$ \\
\hline Observations & 243 & 243 & 243 & 243 & 243 & 243 \\
\hline R-squared & 0.16715 & 0.22449 & 0.23670 & 0.21385 & 0.24787 & 0.26147 \\
\hline
\end{tabular}

Notes: Robust standard errors in parentheses; *** $p<0.01$, ** $p<0.05$, * $p<0.1$ 


\section{$31 \quad$ BOK Working Paper No.2015-8 (2015.3)}

Table 12: Post-Crisis Estimates

\begin{tabular}{|c|c|c|c|c|c|c|}
\hline \multirow{2}{*}{ VARIABLES } & (1) & (2) & (3) & (4) & (5) & (6) \\
\hline & sreturns3 & sreturns3 & sreturns3 & freturn3 & freturn3 & freturn3 \\
\hline \multirow[t]{2}{*}{ Futures Foreign } & $0.01289^{* * *}$ & & $0.00598^{* * *}$ & $0.01574^{* * *}$ & & $0.00799^{* * *}$ \\
\hline & $(0.00170)$ & & $(0.00199)$ & $(0.00187)$ & & $(0.00216)$ \\
\hline \multirow[t]{2}{*}{ Lag Futures Foreign } & $-0.00231^{*}$ & & -0.00129 & $-0.00366^{* *}$ & & -0.00228 \\
\hline & $(0.00138)$ & & $(0.00150)$ & $(0.00154)$ & & $(0.00167)$ \\
\hline \multirow[t]{2}{*}{ Spot Foreign } & 0.00135 & & $0.00262^{* *}$ & 0.00207 & & $0.00349^{* *}$ \\
\hline & $(0.00117)$ & & $(0.00127)$ & $(0.00140)$ & & $(0.00142)$ \\
\hline \multirow[t]{2}{*}{ Lag Spot Foreign } & -0.00149 & & -0.00181 & -0.00147 & & -0.00189 \\
\hline & $(0.00134)$ & & $(0.00133)$ & $(0.00149)$ & & $(0.00147)$ \\
\hline \multirow[t]{2}{*}{ Futures DFls } & & $-0.00660^{* * *}$ & $0.04708^{* * *}$ & & $-0.00871^{* * *}$ & $0.05548^{* * *}$ \\
\hline & & $(0.00199)$ & $(0.01539)$ & & $(0.00217)$ & $(0.01730)$ \\
\hline \multirow[t]{2}{*}{ Lag Futures DFls } & & 0.00080 & 0.02041 & & 0.00172 & 0.01942 \\
\hline & & $(0.00150)$ & $(0.01618)$ & & $(0.00167)$ & $(0.01706)$ \\
\hline \multirow[t]{2}{*}{ Spot DFls } & & $-0.00652^{* * *}$ & $-0.00644^{* * *}$ & & $-0.00724^{* * *}$ & $-0.00716^{* * *}$ \\
\hline & & $(0.00091)$ & $(0.00093)$ & & $(0.00100)$ & $(0.00101)$ \\
\hline \multirow[t]{2}{*}{ Lag Spot DFls } & & 0.00102 & $0.00132^{*}$ & & $0.00141^{*}$ & $0.00172^{* *}$ \\
\hline & & $(0.00075)$ & $(0.00073)$ & & $(0.00083)$ & $(0.00083)$ \\
\hline \multirow[t]{2}{*}{ Lag Cross Market Returns } & -0.02168 & -0.03110 & -0.04225 & -0.03693 & -0.04550 & -0.05952 \\
\hline & $(0.03417)$ & $(0.03421)$ & $(0.03313)$ & $(0.04367)$ & $(0.04391)$ & $(0.04327)$ \\
\hline \multirow[t]{2}{*}{ Constant } & 0.00382 & $0.01390^{* * *}$ & $0.01359^{* * *}$ & $0.00772^{*}$ & $0.01907^{* * *}$ & $0.01809^{* * *}$ \\
\hline & $(0.00382)$ & $(0.00413)$ & $(0.00428)$ & $(0.00428)$ & $(0.00465)$ & $(0.00478)$ \\
\hline Observations & 859 & 859 & 859 & 859 & 859 & 859 \\
\hline R-squared & 0.08984 & 0.16544 & 0.19006 & 0.10317 & 0.17379 & 0.20137 \\
\hline
\end{tabular}

Notes: Robust standard errors in parentheses; $* * * \quad p<0.01, * * p<0.05, * p<0.1$

Table 13: Post-Crisis Estimates (Expected and Unexpected)

\begin{tabular}{|c|c|c|c|c|c|c|}
\hline \multirow{2}{*}{ VARIABLES } & $(1)$ & (2) & (3) & $(4)$ & (5) & $(6)$ \\
\hline & sreturns3 & sreturns3 & sreturns3 & freturn3 & freturn3 & freturn3 \\
\hline \multirow[t]{2}{*}{ Unexpected Futures Foreign } & $0.01343^{* * *}$ & & $0.00637^{* *}$ & $0.01626^{* * *}$ & & $0.00855^{* * *}$ \\
\hline & $(0.00207)$ & & $(0.00251)$ & $(0.00226)$ & & $(0.00271)$ \\
\hline \multirow[t]{2}{*}{ Lag Unexpected Futures Foreign } & $-0.00720^{*}$ & & -0.00226 & $-0.01237^{* * *}$ & & $-0.00840^{*}$ \\
\hline & $(0.00380)$ & & $(0.00421)$ & $(0.00422)$ & & $(0.00458)$ \\
\hline \multirow[t]{2}{*}{ Expected Futures Foreign } & $0.02808^{* *}$ & & 0.01135 & $0.04334^{* * *}$ & & $0.02963^{* *}$ \\
\hline & $(0.01149)$ & & $(0.01278)$ & $(0.01278)$ & & $(0.01397)$ \\
\hline \multirow[t]{2}{*}{ Lag Expected Futures Foreign } & -0.01429 & & -0.00763 & $-0.02417^{* *}$ & & $-0.01963^{*}$ \\
\hline & $(0.00886)$ & & $(0.00935)$ & $(0.00990)$ & & $(0.01026)$ \\
\hline \multirow[t]{2}{*}{ Unexpected Spot Foreign } & 0.00225 & & 0.00290 & $0.00347^{* *}$ & & $0.00429^{* *}$ \\
\hline & $(0.00149)$ & & $(0.00183)$ & $(0.00176)$ & & $(0.00207)$ \\
\hline \multirow[t]{2}{*}{ Lag Unexpected Spot Foreign } & 0.00357 & & 0.00225 & 0.00297 & & 0.00124 \\
\hline & $(0.00220)$ & & $(0.00225)$ & $(0.00249)$ & & $(0.00253)$ \\
\hline \multirow[t]{2}{*}{ Expected Spot Foreign } & $-0.02250^{*}$ & & -0.01085 & -0.01749 & & -0.00384 \\
\hline & $(0.01345)$ & & $(0.01440)$ & $(0.01494)$ & & $(0.01594)$ \\
\hline \multirow[t]{2}{*}{ Lag Expected Spot Foreign } & 0.00861 & & 0.00029 & 0.00444 & & -0.00491 \\
\hline & $(0.01015)$ & & $(0.00989)$ & $(0.01150)$ & & $(0.01094)$ \\
\hline \multirow[t]{2}{*}{ Unexpected Futures DFls } & & $-0.00728^{* * *}$ & $0.05042^{* * *}$ & & $-0.00937^{* * * *}$ & $0.05345^{* * *}$ \\
\hline & & $(0.00250)$ & $(0.01569)$ & & $(0.00270)$ & $(0.01790)$ \\
\hline \multirow[t]{2}{*}{ Lag Unexpected Futures DFls } & & 0.00387 & 0.03263 & & $0.00864^{* *}$ & 0.04254 \\
\hline & & $(0.00385)$ & $(0.02716)$ & & $(0.00418)$ & $(0.03128)$ \\
\hline
\end{tabular}


(Table 13, continued)

\begin{tabular}{|c|c|c|c|c|c|c|}
\hline \multirow{2}{*}{ VARIABLES } & (1) & (2) & (3) & (4) & (5) & (6) \\
\hline & sreturns3 & sreturns3 & sreturns3 & freturn3 & freturn3 & freturn3 \\
\hline Expected Futures DFls & & $\begin{array}{l}-0.01755 \\
(0.01097)\end{array}$ & $\begin{array}{l}0.06854 \\
(0.06942)\end{array}$ & & $\begin{array}{l}-0.03256^{* * *} \\
(0.01206)\end{array}$ & $\begin{array}{l}0.02153 \\
(0.08224)\end{array}$ \\
\hline Lag Expected Futures DFls & & $\begin{array}{l}0.01358^{*} \\
(0.00824)\end{array}$ & $\begin{array}{l}0.05536 \\
(0.06638)\end{array}$ & & $\begin{array}{l}0.02371^{* * *} \\
(0.00898)\end{array}$ & $\begin{array}{l}0.06659 \\
(0.07779)\end{array}$ \\
\hline Unexpected Spot DFls & & $\begin{array}{l}-0.000000^{\text {etw }} \\
(0.00106)\end{array}$ & $\begin{array}{l}-0.00002^{* * *} \\
(0.00109)\end{array}$ & & $\begin{array}{l}-0.00664^{* * * *} \\
(0.00115)\end{array}$ & $\begin{array}{l}-0.00669^{* * *} \\
(0.00117)\end{array}$ \\
\hline Lag Unexpected Spot DFls & & $\begin{array}{l}0.00268^{* *} \\
(0.00107)\end{array}$ & $\begin{array}{l}0.00235^{* *} \\
(0.00113)\end{array}$ & & $\begin{array}{l}0.00299^{* *} \\
(0.00119)\end{array}$ & $\begin{array}{l}0.00277^{* *} \\
(0.00124)\end{array}$ \\
\hline Expected Spot DFls & & $\begin{array}{l}-0.02613^{* * *} \\
(0.00843)\end{array}$ & $\begin{array}{l}-0.02196 * * \\
(0.00929)\end{array}$ & & $\begin{array}{l}-0.02455^{* * *} \\
(0.00933)\end{array}$ & $\begin{array}{l}-0.02138^{* *} \\
(0.01005)\end{array}$ \\
\hline Lag Expected Spot DFls & & $\begin{array}{l}0.01364^{*} \\
(0.00750)\end{array}$ & $\begin{array}{l}0.01131 \\
(0.00858)\end{array}$ & & $\begin{array}{l}0.01174 \\
(0.00830)\end{array}$ & $\begin{array}{l}0.01015 \\
(0.00916)\end{array}$ \\
\hline Lag Cross Market Returns & $\begin{array}{l}0.04906 \\
(0.04677)\end{array}$ & $\begin{array}{l}-0.02726 \\
(0.04714)\end{array}$ & $\begin{array}{l}-0.04295 \\
(0.05210)\end{array}$ & $\begin{array}{l}0.06957 \\
(0.05863)\end{array}$ & $\begin{array}{l}-0.00301 \\
(0.05888)\end{array}$ & $\begin{array}{l}-0.01981 \\
(0.06504)\end{array}$ \\
\hline Constant & $\begin{array}{l}0.01686 \\
(0.01189)\end{array}$ & $\begin{array}{l}0.02791^{* * *} \\
(0.00807)\end{array}$ & $\begin{array}{l}0.03406^{* * *} \\
(0.01254)\end{array}$ & $\begin{array}{l}0.02112 \\
(0.01370)\end{array}$ & $\begin{array}{l}0.03322^{* * *} \\
(0.00927)\end{array}$ & $\begin{array}{l}0.03812^{* * *} \\
(0.01427)\end{array}$ \\
\hline Observations & 676 & 676 & 676 & 676 & 676 & 676 \\
\hline R-squared & 0.11216 & 0.18809 & 0.21397 & 0.12992 & 0.19921 & 0.22587 \\
\hline
\end{tabular}

Notes: Robust standard errors in parentheses; $* * * p<0.01, * * p<0.05, \quad * p<0.1$

Fifth, across subgroups of DFI s19), different groups show different trading patterns in both markets. In futures markets, apart from the significant effect of foreigners' net positions, net positions of asset management funds and DFI s played an important role in pricing with independent trading decisions. Banks and securities companies seem to react to foreign investors' trading, as their coefficients are significant only when DFI subgroups are run, but their effects disappear if foreign trading is induded. In futures markets, domestic securities companies account for the largest share (about 30\% in 2004 and 60\% in 2013), while banks are also sizeable.20) But when compared to the foreign investors,21) DFIs are fragmented; that is, they consist of a large number of smaller accounts, which

19) We have done the same analysis for disaggregated DFIs by investor subgroups for the full sample period, but we don't show here the results (Tables). The empirical results can be available upon request.

20) Banks have hedging demand for their cash bond holdings and are likely to take a large short positions in the futures market based on a similar market view through relatively long-term market analysis; similarly, domestic securities companies may take a short positions for hedging.

21) Usually, it is only several large foreign investors who make very large investments and drive market prices. 
dilutes their collective influence on pricing. This could explain the weaker price effects of banks and security companies in the futures market compared to foreigners. In contrast, banks and funds appear to be dominant players in the cash markets, and their transactions have a significant impact on cash prices. In addition, the pricing effects of different DFI subgroups are different for bond tenors.

As characterizing several different aspects of such empirical analysis, its features are as follows: 1) Investors' net long positions in the futures market have significantly affected the return movements in the cash market and especially, foreign investors appear to be more influential in both futures and cash markets than are domestic investors; 2) The effects of contemporaneous and lagged net purchases and net long positions on returns show the opposite sign since investors rely on lagged information when making current investment decisions, and foreign investors' net long positions in the futures market has a significant influence on cash and futures returns whereas their net purchases in the cash market does not; 3) The expected components of net long positions of foreign investors in the futures market and net purchases of domestic investors in the cash market have a greater effect on returns than do the unexpected components. On the other hand, foreign investors are likely to be better informed and, therefore, the unexpected components of their net long positions in futures market have a relatively greater influence than do domestic investors; 4) The effect of lagged cross-market returns is significant in the pre-crisis period and gradually disappears after the crisis, seemingly for the reason that investors turn to other factors than lagged cross-market returns when making decisions; 5) As for the subgroups of domestic investors in the futures market, asset management and funds play an important role in pricing as independent entities while banks and security firms act as dealers. In the cash market, however, banks and funds are rather significantly influential in pricing. Although domestic banks and security firms account for a much larger share in futures trading than do foreign investors, they seem less influential in the market because of their fragmented purchases and low priaing power. 


\section{Summary and Policy Implications}

This paper sheds light on the role of foreign investors in price discovery through their participation in KTBs and their futures markets. We find that the futures market leads price discovery in the Korean bonds markets. The model fit for the price effects of trading improves substantially if trading in both cash and futures markets is induded, as opposed to considering only one of these two markets. Furthermore, the futures market contributes significantly to price movement in the cash market, while the converse is not true. This finding is in line with previous literature for advanced economies such as the US and Canada.

Our results also suggest that foreign investors' net long futures positions drive price discovery in the KTB cash and futures markets. These exert significant influence in cash and futures returns, but their net purchases in the cash market do not. The trading behavior of foreign investors participating in the futures market also appears to be different from that of foreign investors in the cash market. As for DFIs, their net cash purchases impact both cash and futures returns. Although the effects of DFI net positions on the futures returns are also significant, these seem to be explained largely by a mirror-effect of foreigners' trading, as DFIs take the offsetting positions for the foreign investors' net positions in the futures market.

Empirical results also suggest that the pricing effects of how foreign and domestic investors trade are different. Compared to DFIs, foreign net futures positions are more unpredictable, driven by factors beyond the usual domestic and global economic conditions, such as their indination to herding and speculation and other private information. On the other hand, DFI trading decisions in the 3-year cash market are more predictable and based on overall economic conditions (expected).

In terms of the pricing effects of their transactions, both expected and unexpected components exert significant influence on bond prices. Although the expected component of both investor groups trading decisions appears to be more important for pricing than the unexpected, there is a difference in the degree of pricing effects of how foreign and domestic investors trade, based on their responses 
to news and private information. That is, for DFIs, the expected component weighs much more than the unexpected in pricing effect of their cash trading, while for foreign investors the unexpected component plays an important role comparable to the role of the expected component in the pricing effect of their futures trading.

Overall, our findings suggest that foreign participation in KTB futures has been useful for price discovery by helping investors exploit extra information from their trading behaviors, whether this additional information is based on their response to global and local news or private information. It also appears that both foreign investors and DFI shave improved information gathering and sharing through their trading. However, our findings also suggest that the price effect of foreign net futures positions is significant and affected by the unexpected component of their trading. To the extent that the type of foreign investors entering the futures market is different from the one in the cash market (that is, potentially more speculative and indined to herding behavior), the possibility of a destabilizing effect of foreign participation in the futures market cannot be overlooked. Further research is warranted to understand the motivations behind foreign investors' participation in futures market based on their types and how these are related to market volatility.

The main features of the pre-crisis, crisis, and post-crisis periods are as follows: The effect of lagged cross-market returns on cash and futures returns is significant in the pre-crisis period, however, gradually disappears during the crisis and throughout the post-crisis period. It owes to that in the post-crisis period information transmission has become almost instant in intraday trading and thus the lagged returns no longer affect cash and futures returns. Also, foreign investors net long positions in the futures market happen to be more significant during the crisis compared to in the pre or post-crisis periods and the effect of domestic investors' net purchases and net long positions in the cash and futures markets have become significant after the crisis as their trading volume has grown considerably and become more sophisticated. At last, the effect of foreign investors in the cash and futures markets is significant over the entire sample period, but greater during the crisis period at a higher significance level, and the expected components are more influential than are the unexpected.

A few policy implications can be drawn from our study. Price discovery seems to 
improve as the futures market develops; indeed, the Korean experience well illustrates how information transmission through trading can become more efficient in emerging market bond markets over the course of futures market development. However, Korea's experience suggests that foreign investors' trading decisions may be driven by factors that are very different from those driving domestic investors and potentially less predictable. These factors may indude motivations for portfolio rebalancing, private information, idiosyncratic responses to new information, and herding. Nevertheless, the unexpected component of foreign participation in the futures market is shown to have a significant price impact on the local currency bonds. This suggests there may be merit in making more effort to understand what types of foreign investors enter into futures markets especially in developing economies. Different types of investors with different investment strategies and motivations - regardless of their nationalities will likely bring in diversity, adding market liquidity and stability in the long run. However in emerging markets where the market remains small and shallow, foreign investors - especially if their investment strategies and motivations are similar as a group - could have disproportionately large impact on market liquidity and volatility. This rather unpredictable, but common as a group, trading strategy of any certain investor group could have implications for sudden price movement and market instability. Therefore, promoting participation of diverse foreign investor groups and increasing the size and sophistication of domestic investors can help enhance market resilience and stability in emerging local currency bond markets. 


\section{References}

Balduzzi, P. E. Elton, and T. C. Green (2001), "Economic News and Bond Prices:

Evidence from the U.S. Treasury Market," Journal of Financial and Quantitative Analysis, Vol. 36, I ssue 4, pp. 523-543.

Bessembinder, H., and P. Seguin (1993), "Price Volatility, Trading Volume, and Market Depth: Evidence from Futures Markets," Journal of Financial and Quantitative Analysis, Vol. 26, I ssue 1, pp. 21-39.

Brandt, M. K. Kavajec, and S. Underwood (2007), "Price Discovery in the Treasury Futures Market," The Journal of Futures Market, Vol. 27, I ssue 11, pp. 1021-1051.

Burger J., F. Warnock, and V. C. Warnock (2012), “Emerging Local Currency Bond Markets," Financial AnalystJournal, Vol. 68, No. 4, pp. 73-93.

Burger J., and F. Warnock (2007), "Foreign Participation in Local Currency Bond Markets," Review of Financial Economics, Vol. 16, I ssue 3, pp. 291-304.

Campbell, B., and S. Hendry (2007), "Price Discovery in Canadian and U.S. 10-Year Government Bonds," Bank of Canada Working Paper 2007-43.

Fleming, J., B. Ostdiek, and R. E. Whaley (1996), "Trading Costs and the Relative Rates of Price Discovery in Stocks, Futures, and Options Markets," The Journal of Futures Markets, Vol. 16, I ssue 4, pp. 353-387.

Girardi, A., And C. I mpenna (2013), "Price Discovery in the I talian Treasury Bonds Market: The Role of Order Flow," Temi di DiscussioneNo. 906.

Hautsch, N., and D. Hess (2007), "Bayesian Learning in Financial Markets: Testing for the Relevance of I nformation Precision in Price Discovery," Journal of Financial and Quantitative Analysis, Vol. 42, I ssue 1, pp. 189-208.

Huang, M.-W., and H. Zhang (1995), "Price Movements and Price Discovery in the Municipal Bond Index and the Index Futures Markets," The Journal of FuturesMarkets, Vol. 15, I ssue 4, pp. 489-506.

International Monetary Fund (IMF) (2005), "Development of Corporate Bond Markets in Emerging Market Countries," Global Financial Stability Report September 2005. 
J iang, G., I. Lo, and A. Verdelhan (2011), "I nformation Shocks, Liquidity Shocks, Jumps, and Price Discovery: Evidence from the U.S. Treasury Market," Journal of Financial and Quantitative Analysis, Vol. 46, I ssue 2, pp. 527-551.

Kim, M., A. Szakmary, and T. Schwarz (1999), "Trading Cost and Price Discovery Across Stock Index Futures and Cash Markets," The Journal of Futures Markets, Vol. 19, I ssue 4, pp. 475-498.

Peiris, S. (2010), “Foreign Participation in Emerging Markets' Local Currency Bond Markets," International Monetary Fund Working Paper No. WP/10/88.

Richards, A. (2005), "Big Fish in Small Ponds: The Trading Behavior and Price Impact of Foreign I nvestors in Asian Emerging Equity Markets," Journal of Financial and Quantitative Analysis, Vol. 40, I ssue 1, pp. 1-27.

Roldos, J. (2004), "Emerging Local Bond Markets," Emerging Local Securities and Derivatives Markets, World Economic and Financial Surveys, International Monetary Fund.

Simpson, W. G., and T. I reland (1985), "The I mpact of Finandial Futures on the

Cash Market for Treasury Bills," Journal of Financial and Quantitative Analysis, Vol. 20, Issue 3, pp. 371-379.

Theissen, E. (2012), "Price Discovery in the Spot and Futures Markets: A Reconsideration," The European Journal of Finance, Vol. 18, I ssue 10, pp. 969-987.

Witherspoon, J. (1993), "How Price Discovery by Futures Impact the Cash Market," The Journal of Futures Market, Vol. 13, Issue 5, pp. 469-496.

Zhong, M., A. F. Darrat, and R. Otero (2004), "Price Discovery and Volatility Spillovers in I ndex Futures Markets: Some Evidence from Mexico," J Journal of Banking and Finance, Vol. 28, I ssue 12, pp. 3037-3054. 


\section{$<$ Abstract in Korean $>$}

\section{최재훈*, 임호성**, Rogelio Jr. MERCADO***, Cyn-Young PARK****}

본고는 한국거래소의 국채선물 및 장외시장에서의 국채현물 일별 통계를 활용하여 국채 현·선물시장에서의 외국인 참여의 영향과 가격결정과정(price discovery)에서의 역할을 분석하였다.

분석 결과 외국인의 국채선물시장 참여는 국채현물시장에서의 가격결정과정 을 선도하는 것으로 나타났다. 특히 외국인은 글로벌 뉴스나 사적 정보 등을 활용하여 거래에 참여하기 때문에 국내 투자자들의 정보 탐색에 도움을 줌으로써 가격결정과정에 기여한 것으로 나타났다.

실증분석에 따르면 국채선물시장에서의 외국인 일별 순매수 포지션은 국채선물과 국채현물 가격에 유의한 영향을 미치는 것으로 나타난 반면 외국인의 현물 순매수 포지션은 국채 현·선물가격에 유의한 영향을 미치지 못하였다.

또한 외국인의 국채선물 순매수에 따른 가격변화의 일정 부분을 예측되지 않은 순매수 포지션(unexpected component)이 설명하는 것으로 나타났으며 이는 국내 기관들이 획득할 수 없는 추가적인 정보 사항(information content)을 담고 있는 뉴스, 사적 정보 등을 외국인이 순매수 결정시 활용하고 있음을 보여준다.

\footnotetext{
* 한국은행 차장 (gih4423@bok.or.kr)

** 한국은행 전문연구원 (hosung@bok.or.kr)

*** Graduate Student/Research Associate, Trinity College Dublin (mercador@tcd.ie)

**** Director, Asian Development Bank (cypark@adb.org)
} 